\title{
DYNAMICS AND STABILITY OF MICRO-CUTTING OPERATIONS
}

\author{
Hyung Suk Yoon, Kornel F. Ehmann" \\ Department of Mechanical Engineering, Northwestern University, Evanston, IL \\ ${ }^{*}$ Email: k-ehmann@northwestern.edu Tel: 1-847-491-3263, Fax: 1-847-491-3915
}

\begin{abstract}
In spite of considerable advances toward the understanding of the mechanics of microcutting operations their dynamics and stability that are dominated by the influence of nonlinear effects imposed by the minimum chip thickness and grain size effects and the dominance of ploughing over shearing still remain largely uncharted. Hence, the objective of this paper is to explore the fundamental mechanisms that lead to instabilities in micro-cutting. For dynamic stability analysis, linear stability theory was adopted to model regenerative chatter. The rationale for using linear stability theory is to perform a rigorous parametric study of the major factors that influence stability in light of the nonlinear phenomena that dominate micro-cutting processes. A linearized micro-cutting dynamic force model and a linear structural dynamics model are presented to formulate stability criteria for the different characteristic operating regimes encountered in micro-cutting. It has been shown that the three physical operating regimes, pure ploughing, simultaneous ploughing and shearing and the shearing dominant, govern the microcutting process. They are primarily a function of the relationship between the undeformed chip thickness and cutting edge radius and exert different influences on process stability. Based on the linearized cutting dynamics models characterizing these regimes, a generalized approach to dealing with nonlinear force effects was proposed for process stability analysis. The method is applicable for cutting process nonlinearities at all scales if they are present.
\end{abstract}

Need to add conclusion and contribution in the abstract

Keywords: Micro-cutting; Minimum chip-thickness effect; Nonlinear cutting force; Dynamic stability; 


\section{Introduction}

Micro-manufacturing, the creation of high-precision three dimensional products that require the use of a variety of materials and possessing methods with sizes ranging from tens of micrometers to a few millimeters [1], is emerging as one of the leading technologies of the future. It has become an essential technology needed to satisfy industrial demands in almost all industrial sectors. A variety of manufacturing processes were developed and are still being developed, starting from the late eighties, spurred by advances in Micro-Electro-Mechanical Systems (MEMS) science and technologies which are based on semi-conductor processing methods. Despite of the successful utilization of MEMS technologies for micro-fabrication, the growing needs dictated by the use of various materials with more complex shapes led to the development of a much broader range of micro-manufacturing process, some of which were derived from their conventional macro-scale counterparts. LIGA, laser and ion beam machining, micro-ultrasonic machining, micro-EDM (electro-discharge machining,) and micro-ECM (electro-chemical machining) among others were developed and the possibilities to utilize them as micro-fabrication technologies that ensure the desired precision and productivity were examined.

Micro-cutting/machining processes, which are rooted in conventional cutting processes, e.g., micro-turning, -milling, -drilling, etc., are rapidly gaining increased significance. The most salient advantages of micro-cutting processes over other processes are their capability to produce three-dimensional complex component geometries in a wide variety of materials. In addition, these processes cover the dimensional range of machined parts from a few hundred $\mu \mathrm{m}$ to a few $\mathrm{mm}$ and bridge the gap between the conventional macro-scale and the nano/micro-scale domains. For these reasons, micro-cutting processes exhibit highly prominent characteristics and the further development of these processes is becoming an increasingly important research topic.

The mechanics of cutting in the micro-scale range cannot be understood by simply scalingdown the understanding of the conventional processes. One of the principal difficulties is related to the edge radius effect, previously referred to as the size effect [2], that is due to the fact that the sharpness of the tool, expressed by the tool-edge radius, cannot be proportionally scaled down. As a consequence, the dimension of the undeformed chip thickness in micro-scale machining is comparable to, or even smaller than, the tool-edge radius leading to fundamentally different cutting mechanisms and the well-established minimum chip thickness related 
phenomena. This leads, in many cases, to the dominance of ploughing over shearing [3]. One of the consequences of this change in the cutting mechanism in micro-cutting is that, unlike for conventional cutting processes where the process can be described by a linear function of the undeformed chip thickness and the ploughing mechanisms can be neglected, the micro-cutting process and, in particular, its dynamics cannot be described by linear models due to the nonlinear change in the cutting force as a function of the undeformed chip thickness. Similarly, the friction force at the clearance face, due to the elastic recovery of the work material, that is generally negligible in conventional cutting, cannot be neglected either in micro-cutting and constitutes an additional nonlinear factor [4]. Moreover, due to the small values of the feed per tooth that can be equal or smaller than the grain size of the material being cut another nonlinear behavior comes into play. Under such circumstances the assumption that the work material is a continuum is no longer valid and the microstructure of the work material and its effects on the process need to be also taken into account.

In spite of considerable advances toward the understanding of the mechanics of microcutting operations, as summarized in Liu, et al. [4], their stability and, in particular, the influence of the nonlinear effects imposed by the above-mentioned mechanisms still remains largely uncharted [5]. There are no explicit relations between the dynamic instability of the process and the effects of the minimum chip thickness, one of the dominant factors in micro-cutting, reported in the literature.

There is a huge body of work related to the stability of conventional cutting operations originating from the pioneering works of Tobias, Tlusty et al. and Merrit [6-8]. Most of these investigations were conducted under the assumption of linear process models. The subsequent works, representing refinements of the basic concepts initially established by the aforementioned researchers generally retained the same assumptions, i.e., the linearity of both the process and the structure and orthogonal cutting conditions, e.g., Smith and Tlusty [9], Minis and Yanushevsky [10, 11], Altintas and Budak [12] and others. However, in real systems, nonlinearity has always been recognized as an essential element in machining as pointed out very early by Hanna and Tobias [13]. Moon, et al. [14] listed the nonlinear phenomena in cutting that require more complex models than the simple linear stability models and categorized the sources of nonlinearity, while Stépán [15] has presented a linearization method to deal with the nonlinearity of the cutting force. 
Regarding the dynamic stability of micro-cutting processes, the technical literature is very sparse. Liu, et al. [4] and Jun, et al. [5] explicitly analyzed the dynamic behavior of the tool and its effect on the cutting force. In addition, dynamic instability at low feed rates, the effect of the minimum chip thickness and elastic recovery were reported by Jun, et al. [16]. However, their focus was on the forced vibrations of the milling tool itself, not on the process dynamics resulting in self-excited vibrations during micro-milling, hence the interaction between process and structural dynamics was not explicitly addressed. Kim et al. [7], in turn, investigated stability in micro-milling with a rounded tool. Again, however, no rigorous model for identifying selfexcited vibrations during the micro-cutting process was established.

In light of the above, the major objective of this paper is to offer a more systematic insight into the fundamental mechanisms that govern the dynamics of micro-cutting processes as well as of their stability under the influence of nonlinear phenomena. The basis for this will be a newly developed nonlinear orthogonal micro-cutting force model that accounts for the minimum chip thickness related effects [18, 19]. Investigations of the dynamic stability of micro-cutting processes will follow. The approach will be based on the linearized form of the force model in conjunction with linear stability analysis. The dynamic stability of the process caused by the minimum chip thickness effect will be described and stability criteria proposed. Stable ranges, corresponding to characteristic micro-cutting conditions and hence different dominant physical cutting mechanisms will be investigated based on the proposed criteria.

Section 2 of the paper gives a quick overview of the micro-cutting model the details of which are reported elsewhere $[18,19]$ that constitutes the basis of this work. The linearization of the model is addressed in Section 3 including an error analysis of the linearized model. The dynamic model and stability criteria are developed in Section 4 based on principles of linear stability theory. A generalized approach to dealing with nonlinear force models to develop the dynamics stability criteria of the micro-cutting process using linear stability theory will be introduced in Section 5 followed by conclusions in Section 6.

\section{Micro-cutting Process Model}

The micro-cutting force model that will be used in the development of a dynamic model of the micro-cutting process is a newly developed formulation based on the slip-line field method. 
The salient feature of this model is the fact that ploughing was not considered as merely rubbing or friction between the work material and the edge of the tool, but instead, as a plastic deformation process of the work material passing beneath the tool. The chip formation process was, in turn, also considered as a plastic deformation process of the work material. Hence, the model considers the influence of both the ploughing and the shearing mechanisms as plastic deformation and flow processes. A schematic representation of the model is given in Fig. 1a. The resulting nonlinear force model was intentionally defined under simple constraints to facilitate its easy linearization for the sole purpose of facilitating a parametric dynamic analysis of the influence of the process' dominant parameters on its stability. For the purposes of the current work it will be briefly summarized below. A detailed account of the complete orthogonal cutting process model that considers the major nonlinear aspects of the process is given in references [18, 19]. The model accounts for the nonlinear components in micro-cutting, namely the ploughing force, the dead metal zone (Fig. 1b), the depth of plastic deformation, and the minimum chip thickness. The dead metal zone, a localized stagnation of the work material at the tool's tip, leads to the modification of the geometry of the plastic deformation zone around the tool tip by modifying the local cutting angles [4].

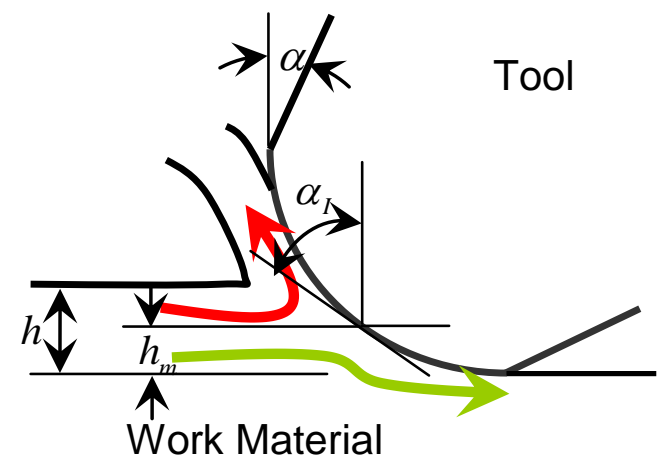

(a)

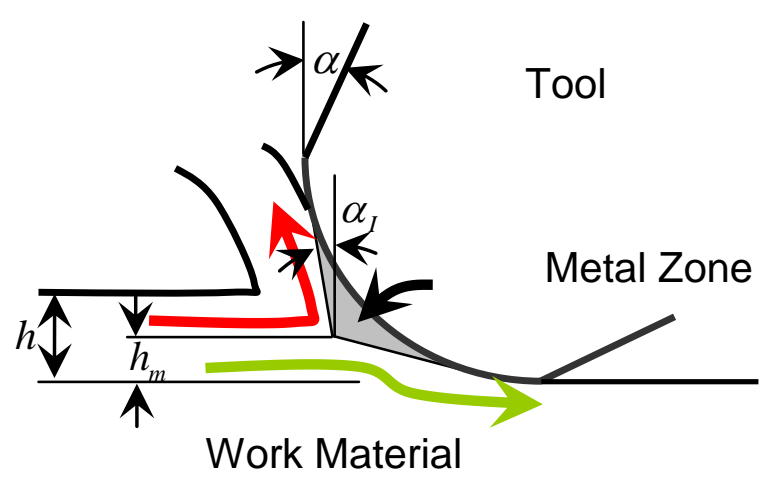

(b)

Fig. 1: Schematics of The Micro-cutting Process:

(a) Without a Dead Metal Zone (b) With a Dead Metal Zone

The model consists of three sets of expressions corresponding to characteristic ranges in the magnitude of the undeformed chip thickness to account for the corresponding different governing physical mechanisms in each of the ranges. The cases that are considered are: 
Case I: $\quad h<h_{m}$

Case II: $\quad h_{m}<h<r_{t}$

Case III: $h>r_{t}$

where

$h: \quad$ The undeformed chip thickness,

$h_{m}: \quad$ The minimum chip thickness, and

$r_{t}: \quad$ The radius of the tool's cutting edge.

Case I takes place when the undeformed chip thickness is smaller than the minimum chip thickness. No chip will be formed and only a ploughing process takes place. In this case, only the plastic deformation process of the material flowing beneath the tool will be modeled. This is referred to as a "pure ploughing process", for there is no other process than ploughing is taking place.

In Case II and Case III, material flow diverges into two paths: one for the chip and the other for the material flowing beneath the tool tip. These two can be described as "chip formation and ploughing process", for the ploughing process takes place simultaneously with the chip formation process in both cases. However, the material flow that forms the chip is different from the conventional machining process in Case II. As the undeformed chip thickness is smaller than the radius of the tool, the machined chip cannot flow on the rake face. The nominal rake angle $\alpha$ has no effect on the actual machining process, for the chip cannot make contact with the rake face due to the geometry of the cut. 
Case III covers the higher undeformed chip thickness range, when the undeformed chip thickness is large enough to make the chip flow on the rake face. The conventional machining process can be categorized into Case III, where the tool radius is much smaller than the undeformed chip thickness.

The micro-cutting force model corresponding to the three above cases is expressed as a multi-variable function of ten variables, i.e., $[18,19]$ :

$$
\begin{gathered}
F_{c}\left\{\begin{array}{l}
{\left[3 \sin \lambda+\left(\frac{\pi}{2}-1-2 \lambda\right) \cdot \cos \lambda-\sqrt{2}\right] \cdot \frac{(1-\cos \rho+\sin \rho)}{\sin \lambda-\cos \lambda} r_{t} \cdot k, h<h_{m}} \\
{\left[(1-\cot (\rho+\gamma)) \cdot\left(h-h_{m}\right)+2 \cdot\left(h_{m}+\delta\right)\right] \cdot k, h_{m}<h<r_{t}} \\
{\left[\left(\cos \phi+\left(1-\frac{\pi}{2}+2 \phi\right) \sin \phi\right) \cdot \frac{\cos \alpha_{I}+\sin \alpha_{I}}{\cos \left(\alpha_{I}-\phi\right)} \cdot\left(h-h_{m}\right)+2\left(h_{m}+\delta\right)\right] \cdot k, \quad h>r_{t}}
\end{array}\right. \\
F_{t}\left\{\begin{array}{l}
{\left[(1-\cot (\rho+\gamma)) \cdot\left(h-h_{m}\right) \cdot\left(1-\frac{\pi}{2}\right)+2 \cdot\left(h-h_{m}+\delta\right)\right] \cdot k, h_{m}<h<r_{t}} \\
{\left[3 \cos \lambda+\left(2 \lambda-\frac{\pi}{2}+1\right) \cdot \sin \lambda-2 \sqrt{2}\right] \cdot \frac{(1-\cos \rho+\sin \rho)}{\sin \lambda-\cos \lambda} r_{t} \cdot k+2 \delta k, h<h_{m}} \\
{\left[\left(2+\left(\left(1-\frac{\pi}{2}+2 \phi\right) \cos \phi-\sin \phi\right) \cdot \frac{\cos \alpha_{I}+\sin \alpha_{I}}{\cos \left(\alpha_{I}-\phi\right)} \cdot\left(h-h_{m}\right)+2 \delta\right] \cdot k, \quad h>r_{t}\right.}
\end{array}\right.
\end{gathered}
$$

where

$\rho: \quad$ The angle of the tangent to the cutting edge at the contact point between the work material and the tool

$\lambda: \quad$ The contact angle in the pure ploughing process

$k: \quad$ The shear strength of the material

$\gamma: \quad$ The flow angle of the primary shear zone

$\delta$ : $\quad$ Depth of plastic deformation

$\alpha_{I}: \quad$ The effective rake angle

$\phi$ : $\quad$ Shear angle.

For the case when $h_{m}<h<r_{t}$, Fig. 2 shows all the above variables, except for $\lambda$ and $\phi$, which are not relevant in this particular case. 


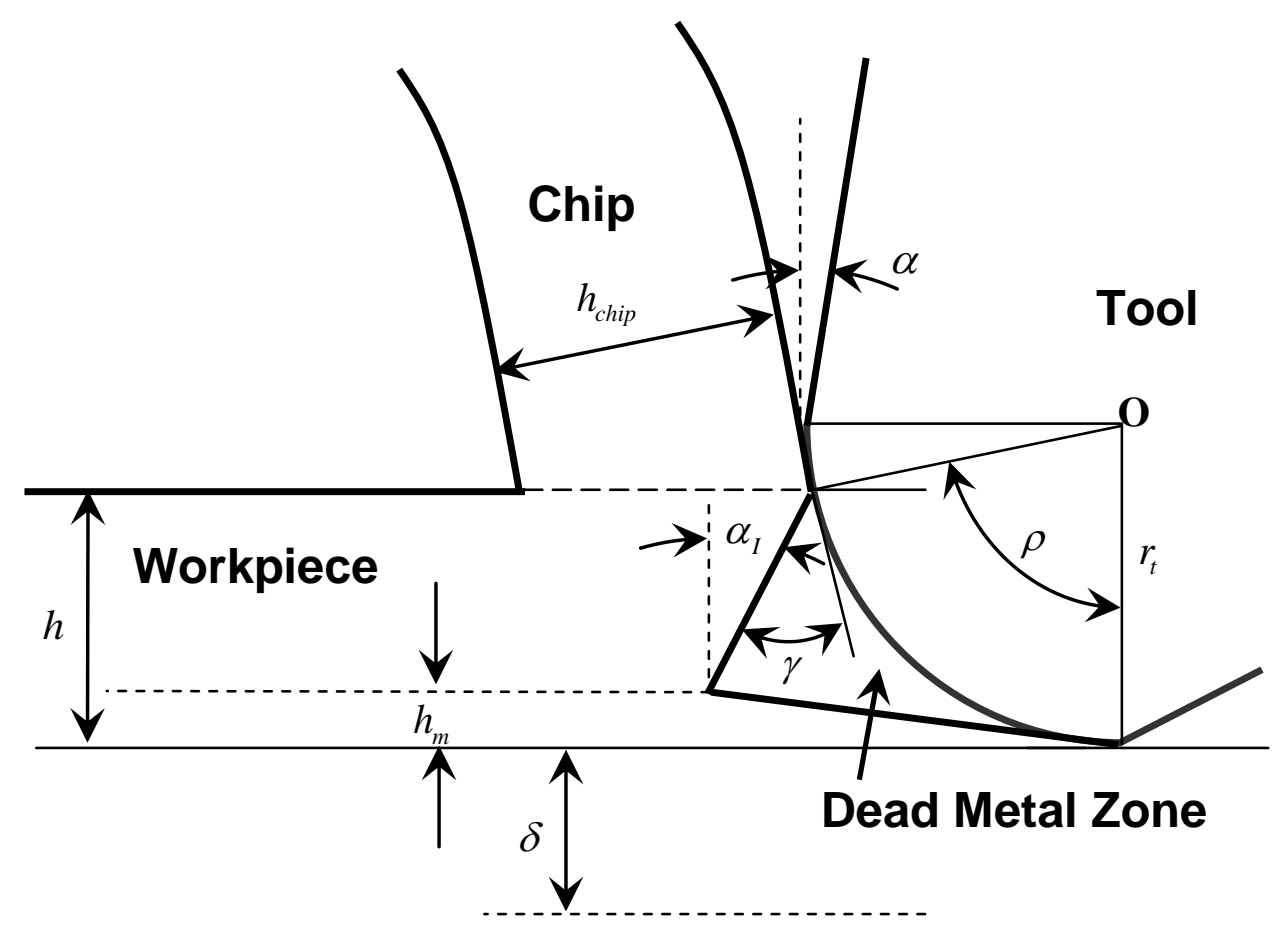

Fig. 2: Micro-cutting Process Variables for the Case $h_{m}<h<r_{t}$

It should be noted that the force model expressed by Eqs. (2a) and (2b) not only contains nonlinear components that are the consequence of the minimum chip thickness and the dead metal zone induced effects, but is also defined in a piecewise manner with discontinuities at points at which a change in the physical mechanisms that govern the micro-cutting process occur. These discontinuities appear at the boundaries expressed by Eq. (1).

As indicated above in Case I, Eq. (1), "pure ploughing" without chip formation is taking place with the plastically deformed material flowing beneath the tool since the undeformed chip thickness is smaller than the minimum chip thickness. When the undeformed chip thickness is larger than the minimum chip thickness value for a given tool edge radius and workpiece material combination "chip formation and ploughing" take place corresponding to cases Cases II and III. The material flow diverges into two flow directions, i.e., one for the chip and the other for the material flowing beneath the tool tip. The principal difference between the two is whether the chip flow does (Case II) of not (Case III) contact the tool's rake face. It should be recognized, that given the geometry of the cutting zone corresponding to these two cases substantially 
different chip flow mechanisms govern the process. In Case II, as the undeformed chip thickness is smaller than the radius of the tool's cutting edge, the chip does not come in contact with the rake face and the nominal rake angle, $\alpha$, has no effect on the process. The material flow that forms the chip is different from that in conventional machining processes. On the other hand, when the undeformed chip thickness is larger than the tool's edge radius, Case III, the undeformed chip thickness is large enough to lead to chip contact and flow on the tool's rake face. Conventional machining processes, in general, correspond to Case III.

It should also be noted that the formation of a dead metal zone in front of the tool tip alters the direction of the metal flow and leads to the modification of the geometry of the plastic deformation zone around the tool tip. The influence of this zone is considered in Cases II and III to offer a more realistic micro-cutting process model. In Case I it is assumed that no dead metal zone will be formed.

\section{Linearization of the Cutting Force Model}

Linear stability theory that will be used for stability analysis in later Sections to deal with the time-delayed effect of regenerative chatter requires a linear force model of the machining process. Therefore, the linearization of the proposed model, which includes nonlinear terms, is the first step in the development of a dynamic model of the micro-cutting process that will facilitate the formulation of stability criteria.

In the previous Section, a cutting force model, which includes nonlinear components stemming from the phenomena associated with micro-cutting processes, was considered. In macro-scale cutting a number of efforts were made to deal with the nonlinear dynamics effects originating from the nonlinear relationship between the stress and strain by several researchers. Among them, Stépán [15] has presented a simple method to deal with the nonlinearity of the cutting force by approximating the nonlinear curve by lines with different slopes as a function of the chip thickness. The slopes of the approximating line at a given value of the chip thickness were considered to play the role of the customary cutting coefficient. However, this approximation method is not suitable for accounting for the effects of the minimum chip thickness, ploughing, change in the depth of plastic deformation, etc. for these effects cannot be separated from the overall cutting force. The utilized linearization method of the cutting force 
function is applicable for functions of a single variable. However, the force models corresponding to the three cases expressed by Eq. (2) are multi-variable functions.

Finding a proper linearization method of the cutting force is the quintessential work that will be addressed in this paper. For multi-variable functions, one of the most commonly used methods of linearization is the Taylor series expansion. The general form of the Taylor series expansion of a function of $n$ variables near a point $X\left(a_{1}, a_{2}, a_{3}, \ldots, a_{n}\right)$ is:

$$
f\left(x_{1}, \ldots, x_{n}\right)=\sum_{j=0}^{\infty}\left\{\frac{1}{j !}\left[\sum_{k=1}^{n}\left(x_{k}-a_{k}\right) \frac{\partial}{\partial x_{k}^{\prime}}\right]^{j} f\left(x_{1}^{\prime}, \ldots, x_{n}^{\prime}\right)\right\}_{x_{1}^{\prime}=a_{1}, \ldots, x_{n}^{\prime}=a_{n}}
$$

If the force expressions, Eq. (2), are linearized near the point $h=h_{o}$ and only the first two terms in the expansion are retained, one obtains:

$$
\begin{aligned}
& F_{c}(h)_{h=h_{o}}=\left[F_{c}\left(h_{0}\right)-\frac{d F_{c}}{d h}\left(h_{0}\right) \cdot h_{0}\right]+\frac{d F_{c}}{d h}\left(h_{0}\right) \cdot h \\
& F_{t}(h)_{h=h_{o}}=\left[F_{t}\left(h_{0}\right)-\frac{d F_{t}}{d h}\left(h_{0}\right) \cdot h_{0}\right]+\frac{d F_{t}}{d h}\left(h_{0}\right) \cdot h
\end{aligned}
$$

Equations (4a) and (4b) indicate that each force component can now be expressed as a sum of a constant term and a term linearly proportional to the chip thickness. To complete the expansion, the first derivatives of force equations should be calculated. This in turn, necessitates the analysis and identification of the independent variables in the model expressed by Eq. (2a) and (2b). As it will be shown, some of the variables are invariant once the tool and the work material are determined/selected. The radius of the tool and the shear strength remain fixed during the machining process and can be regarded as constants.

The minimum chip thickness, $h_{m}$, the flow angle of the primary shear zone, $\gamma$, and the effective rake angle $\alpha_{I}$ can be expressed as $[18,19]$ :

$$
h_{m}=r_{t} \cdot\left(1-\cos \left(\frac{\pi}{4}-\frac{\tan ^{-1} \mu}{2}\right)\right)
$$




$$
\begin{aligned}
& \gamma=\tan ^{-1} \mu . \\
& \alpha_{I}=\tan ^{-1} \mu, \text { for } h>r_{t} .
\end{aligned}
$$

where $\mu$ is the coefficient of friction. As it can be seen, all three variables are a function of the friction coefficient. It is assumed that once the tool and the work material are selected, the contact conditions are invariant resulting in a constant friction coefficient. Therefore, $h_{m}, \gamma$, and $\alpha_{I}$ will be constant. The contact angle in the pure ploughing process is expressed by $[18,19]$ :

$$
\lambda=\rho+\eta
$$

in which $\eta$ remains constant under the assumption of a constant friction coefficient since:

$$
\eta=\tan ^{-1}\left(\frac{1+\mu}{1-\mu}\right)
$$

However, as $\lambda$ is the sum of $\rho$ and of a constant, $\lambda$ cannot be counted as an independent variable.

As a consequence of the above considerations, the independent variables of interest are $\rho, h$, $\delta$, and $\phi$, hence, the force model can be expressed as:

$$
\begin{aligned}
& F_{c}=F_{c}(\rho, h, \delta, \phi) \\
& F_{t}=F_{t}(\rho, h, \delta, \phi)
\end{aligned}
$$

yielding the following general expressions for the first order total derivatives:

$$
\begin{aligned}
& \frac{d F_{c}}{d h}=\frac{\partial F_{c}}{\partial \rho} \cdot \frac{d \rho}{d h}+\frac{\partial F_{c}}{\partial \phi} \cdot \frac{d \phi}{d h}+\frac{\partial F_{c}}{\partial \delta} \cdot \frac{d \delta}{d h}+\frac{\partial F_{c}}{\partial h} \\
& \frac{d F_{t}}{d h}=\frac{\partial F_{t}}{\partial \rho} \cdot \frac{d \rho}{d h}+\frac{\partial F_{t}}{\partial \phi} \cdot \frac{d \phi}{d h}+\frac{\partial F_{t}}{\partial \delta} \cdot \frac{d \delta}{d h}+\frac{\partial F_{t}}{\partial h}
\end{aligned}
$$


As the force equations were defined differently for the three cases, the corresponding linearized equations will be developed accordingly.

\subsection{Case I: $h<h_{m}$}

In the force model for the pure ploughing process, expressed by the first pair of equations in Eq. (2a) and Eq. (2b), the only independent variables are $\rho$ in the first and $\rho$ and $\delta$ in the second equation respectively. Therefore, the total derivatives are:

$$
\begin{aligned}
& \frac{d F_{c, I}}{d h}=\frac{\partial F_{c}}{\partial \rho} \cdot \frac{d \rho}{d h} \\
& \frac{d F_{t, I}}{d h}=\frac{\partial F_{t, I}}{\partial \rho} \cdot \frac{d \rho}{d h}+\frac{\partial F_{t, I}}{\partial \delta} \cdot \frac{d \delta}{d h} .
\end{aligned}
$$

To effectively describe the total derivatives of the proposed force model, each partial derivative term and the derivative of the variable with respect to the undeformed chip thickness are expressed by:

$$
\begin{aligned}
\frac{d \rho}{d h}= & \frac{1}{\sqrt{2 h \cdot r_{t}-h^{2}}}=\mathrm{P} \\
\frac{d \delta}{d \rho}= & \frac{1}{\sqrt{2}} \frac{2 \sin \eta-(\sin \rho+\cos \rho)}{(\sin \lambda-\cos \lambda)^{2}}-\frac{(\cos \lambda+\sin \lambda) \cdot \sin \eta-1}{(\sin \lambda-\cos \lambda)^{2}}=\Gamma \\
\frac{d \delta}{d h}= & \frac{\partial \rho}{\partial h} \cdot \frac{d \delta}{d \rho}=P \cdot \Gamma \\
\frac{\partial F_{c, I}}{\partial \rho}= & {\left[\cos \lambda+\left(1-\frac{\pi}{2}+2 \lambda\right) \cdot \sin \lambda\right] \cdot \frac{(1-\cos \rho+\sin \rho)}{\sin \lambda-\cos \lambda} r_{t} \cdot k } \\
& +\left[3 \sin \lambda+\left(\frac{\pi}{2}-1-2 \lambda\right) \cdot \cos \lambda-\sqrt{2}\right] \cdot \frac{2 \sin \eta-(\sin \rho+\cos \rho)}{(\sin \lambda-\cos \lambda)^{2}} r_{t} \cdot k \\
= & A_{c, I}
\end{aligned}
$$




$$
\begin{aligned}
\frac{\partial F_{t, I}}{\partial \rho} & =\left[-\sin \lambda+\left(2 \lambda-\frac{\pi}{2}+1\right) \cdot \cos \lambda\right] \cdot \frac{(1-\cos \rho+\sin \rho)}{\sin \lambda-\cos \lambda} r_{t} \cdot k \\
& +\left[3 \cos \lambda+\left(2 \lambda-\frac{\pi}{2}+1\right) \cdot \sin \lambda-2 \sqrt{2}\right] \cdot \frac{2 \sin \eta-(\sin \rho+\cos \rho)}{(\sin \lambda-\cos \lambda)^{2}} r_{t} \cdot k \\
& =A_{t, I} \\
\frac{\partial F_{t, I}}{\partial \delta} & =2 r_{t} \cdot k=B_{t, I}
\end{aligned}
$$

Then, Eq. (12a) and (12b) can be expressed in compact form as:

$$
\begin{aligned}
& \frac{d F_{c, I}}{d h}=A_{c, I} \cdot \mathrm{P} \\
& \frac{d F_{t, I}}{d h}=A_{t, I} \cdot \mathrm{P}+B_{t, I} \cdot P \cdot \Gamma .
\end{aligned}
$$

\subsection{Case II: $h_{m}<h<r_{t}$}

The force model in this case is expressed by the second pair of equations in Eq. (2a) and Eq. (2b). As the independent variables in this case are $\rho, \delta$, and $h$, the total derivatives are:

$$
\begin{aligned}
& \frac{d F_{c, I I}}{d h}=\frac{\partial F_{c, I I}}{\partial \rho} \cdot \frac{d \rho}{d h}+\frac{\partial F_{c, I I}}{\partial \delta} \cdot \frac{d \delta}{d h}+\frac{\partial F_{c, I I}}{\partial h} \\
& \frac{d F_{t, I I}}{d h}=\frac{\partial F_{t, I I}}{\partial \rho} \cdot \frac{d \rho}{d h}+\frac{\partial F_{t, I I}}{\partial \delta} \cdot \frac{d \delta}{d h}+\frac{\partial F_{t, I I}}{\partial h} .
\end{aligned}
$$

Note that the depth of plastic deformation, expressed by:

$$
\delta=\frac{\left(h-h_{m}\right) \cdot \tan \alpha_{I}+r_{t} \sin \rho-h_{m}}{2}
$$

is a function of $\rho$ and $h$, hence the derivative $\frac{d \delta}{d h}$ can be evaluated as:

$$
\frac{d \delta}{d h}=\frac{\partial \delta}{\partial \rho} \cdot \frac{d \rho}{d h}+\frac{\partial \delta}{\partial h} .
$$

Consequently, Eq. (20a) and (20b) become: 


$$
\begin{aligned}
& \frac{d F_{c, I I}}{d h}=\frac{\partial F_{c, I I}}{\partial \rho} \cdot \frac{d \rho}{d h}+\frac{\partial F_{c, I I}}{\partial \delta} \cdot\left(\frac{\partial \delta}{\partial \rho} \cdot \frac{d \rho}{d h}+\frac{\partial \delta}{\partial h}\right)+\frac{\partial F_{c, I I}}{\partial h} \\
& \frac{d F_{t, I I}}{d h}=\frac{\partial F_{t, I I}}{\partial \rho} \cdot \frac{d \rho}{d h}+\frac{\partial F_{t, I I}}{\partial \delta} \cdot\left(\frac{\partial \delta}{\partial \rho} \cdot \frac{d \rho}{d h}+\frac{\partial \delta}{\partial h}\right)+\frac{\partial F_{t, I I}}{\partial h}
\end{aligned}
$$

For the evaluation of the total derivatives the corresponding partial derivative terms and the derivatives of the variable with respect to the undeformed chip thickness are expressed as:

$$
\begin{aligned}
& \frac{\partial F_{c, I I}}{\partial \rho}=\frac{1}{\sin ^{2}(\rho+\gamma)} \cdot\left(h-h_{m}\right) \cdot k=A_{c, I I} \\
& \frac{d \rho}{d h}=\frac{1}{\sqrt{2 h \cdot r_{t}-h^{2}}}=\mathrm{P} \\
& \frac{\partial F_{c, I I}}{\partial \delta}=2 k=C_{c, I I} \\
& \frac{\partial \delta}{\partial \rho}=\frac{1}{2}\left(h-h_{m}\right) \cdot \frac{1}{\cos ^{2} \alpha_{I}}+\frac{r_{t}}{2} \cos \rho \\
& \frac{\partial \delta}{\partial h}=\frac{1}{2} \tan \alpha_{I} \\
& \frac{d \delta}{d h}=\left(\frac{1}{2}\left(h-h_{m}\right) \cdot \frac{1}{\cos ^{2} \alpha_{I}}+\frac{r_{t}}{2} \cos \rho\right) \cdot \mathrm{P}+\frac{1}{2} \tan \alpha_{I}=\Delta \\
& \frac{\partial F_{c, I I}}{\partial h}=(1-\cot (\rho+\gamma)) \cdot k=D_{c, I I} \\
& \frac{\partial F_{t, I I}}{\partial \rho}=\frac{1}{\sin ^{2}(\rho+\gamma)} \cdot\left(1-\frac{\pi}{2}\right) \cdot\left(h-h_{m}\right) \cdot k=A_{t, I I} \\
& \frac{\partial F_{t, I I}}{\partial \delta}=2 k=C_{t, I I} \\
& \frac{\partial F_{t, I I}}{\partial h}=(3-\cot (\rho+\gamma)) \cdot k=D_{t, I I}
\end{aligned}
$$

With the above terms, Eq. (20a) and (20b) become: 


$$
\begin{aligned}
& \frac{d F_{c, I I}}{d h}=A_{c, I I} \cdot \mathrm{P}+C_{c, I I} \cdot \Delta+D_{c, I I} \\
& \frac{d F_{t, I I}}{d h}=A_{t, I I} \cdot \mathrm{P}+C_{t, I I} \cdot \Delta+D_{t, I I} .
\end{aligned}
$$

\subsection{Case III: $h>r_{t}$}

The force model in this case is expressed by the third pair of equations in Eq. (2a) and Eq. (2b). The depth of plastic deformation is constant in this case and is given by $[18,19]$ :

$$
\delta=\frac{\left(r_{t}-h_{m}\right) \cdot \tan \alpha_{I}+r_{t}-h_{m}}{2}
$$

and, therefore, the partial derivatives of $\delta$ with respect to the undeformed chip thickness vanish. Consequently, the first order total derivatives of the force equations with respect to the undeformed chip thickness are:

$$
\begin{aligned}
& \frac{d F_{c, I I I}}{d h}=\frac{\partial F_{c, I I I}}{\partial \phi} \cdot \frac{d \phi}{d h}+\frac{\partial F_{c, I I I}}{\partial h} \\
& \frac{d F_{t, I I I}}{d h}=\frac{\partial F_{t, I I I}}{\partial \phi} \cdot \frac{d \phi}{d h}+\frac{\partial F_{t, I I I}}{\partial h} .
\end{aligned}
$$

It can be shown that the relevant total and partial derivatives in this case are:

$$
\begin{aligned}
& \frac{\partial F_{c, I I I}}{\partial \phi}=\frac{\sin \left(2 \phi-\alpha_{I}\right)+\left(1-\frac{\pi}{2}+2 \phi\right) \cos \alpha_{I}}{\cos ^{2}\left(\alpha_{I}-\phi\right)} \cdot\left(\cos \alpha_{I}+\sin \alpha_{I}\right) \cdot\left(h-h_{m}\right) \cdot k=C_{c, I I I} \\
& \frac{d \phi}{d h}=\frac{\left(r_{t}-h_{m}\right) \cdot \tan \alpha_{I}+r_{t}-h_{m}}{\left[\left(r_{t}-h_{m}\right) \cdot \tan \alpha_{I}+\left(h-h_{m}\right)\right]^{2}+\left(h-r_{t}\right)^{2}}=\Phi \\
& \frac{\partial F_{c, I I I}}{\partial h}=\left(\cos \phi+\left(1-\frac{\pi}{2}+2 \phi\right) \sin \phi\right) \cdot \frac{\cos \alpha_{I}+\sin \alpha_{I}}{\cos \left(\alpha_{I}-\phi\right)}=D_{c, I I I}
\end{aligned}
$$




$$
\begin{aligned}
& \frac{\partial F_{t, I I I}}{\partial \phi}=\frac{\cos \left(2 \phi-\alpha_{I}\right)-\left(1-\frac{\pi}{2}+2 \phi\right) \sin \alpha_{I}}{\cos ^{2}\left(\alpha_{I}-\phi\right)} \cdot\left(\cos \alpha_{I}+\sin \alpha_{I}\right) \cdot\left(h-h_{m}\right) \cdot k=C_{t, I I I} \\
& \frac{\partial F_{t, I I I}}{\partial h}=\left(2+\left(\left(1-\frac{\pi}{2}+2 \phi\right) \cos \phi-\sin \phi\right) \cdot \frac{\cos \alpha_{I}+\sin \alpha_{I}}{\cos \left(\alpha_{I}-\phi\right)}\right) \cdot k=D_{t, I I I} .
\end{aligned}
$$

so that Eq. (36a) and (36b) can be represented as:

$$
\begin{aligned}
& \frac{d F_{c, I I I}}{d h}=C_{c, I I I} \cdot \Phi+D_{c, I I I} \\
& \frac{d F_{t, I I I}}{d h}=C_{t, I I I} \cdot \Phi+D_{t, I I I} .
\end{aligned}
$$

\subsection{Evaluation of Linearization Errors}

To validate the linearization, the slopes of the linearized functions were compared to the numerically calculated slopes from the original nonlinear force model, i.e., Eqs, (2a) and (2b). The errors of the linearized functions are quantified and summarized in Table 1. The errors in the table were calculated under the following assumptions: tool cutting edge radius $15 \mu \mathrm{m}$, friction coefficient 0.35 , shear strength of the material set to unity. It can be seen that the linearization errors of the functions range from 0.13 to $4.81 \%$. Also, as shown in $[18,19]$ the linearized functions closely follow the original ones. Note that the source of errors in the linearization is due to the neglecting of the higher order terms in the Taylor series expansion, Eqs. (4a) and (4b). Since the slopes of the linearized functions for both the cutting and the tangential force, as shown, are less than 5\% they are considered sufficiently accurate to be used for the formulation of dynamic stability criteria for the micro-cutting process using linear stability theory. An example for Case II is shown in Fig. 3.

Table 1: Linearization Errors (\%)

\begin{tabular}{|c|c|c|c|}
\cline { 2 - 4 } \multicolumn{1}{c|}{} & Case I & Case II & Case III \\
\hline Cutting Force & 1.35 & 1.68 & 2.16 \\
\hline Tangential Force & 4.81 & 0.32 & 0.13 \\
\hline
\end{tabular}




\section{Dynamics of Micro-cutting Processes}

Regenerative instability, the dominant chatter mechanism in macro-scale cutting operations, has extensively been discussed in the literature, e.g., $[6-8,12]$ and others. The simplest regenerative chatter models consider the time-delay effect in light of the interaction between a simple linear structural dynamics model of the machine tool system and a linear cutting process model. The resulting linear stability models have offered the foundation for understanding the underlying phenomena and for a systematic evaluation of the influence of the controlling parameters on process stability. However, many of the accompanying phenomena that are generally nonlinear in nature including the limit-cycle behavior itself cannot be explained by these models. Many past efforts were made to describe the effects of process nonlinearity on the stability of the machining process through modeling the complex interrelations between its dynamics and thermodynamics [21] as well as the stochastic properties of the material to reflect variations in the workpiece properties, in particular, on the cutting resistance [22]. The current paper describes the influence of nonlinear effects on the dynamic stability in the realm of microcutting operations that are governed by phenomena imposed by the minimum chip thickness effect, ploughing, etc. The models developed in the previous sections have clearly shown the profound nonlinearity of the micro-cutting force. 

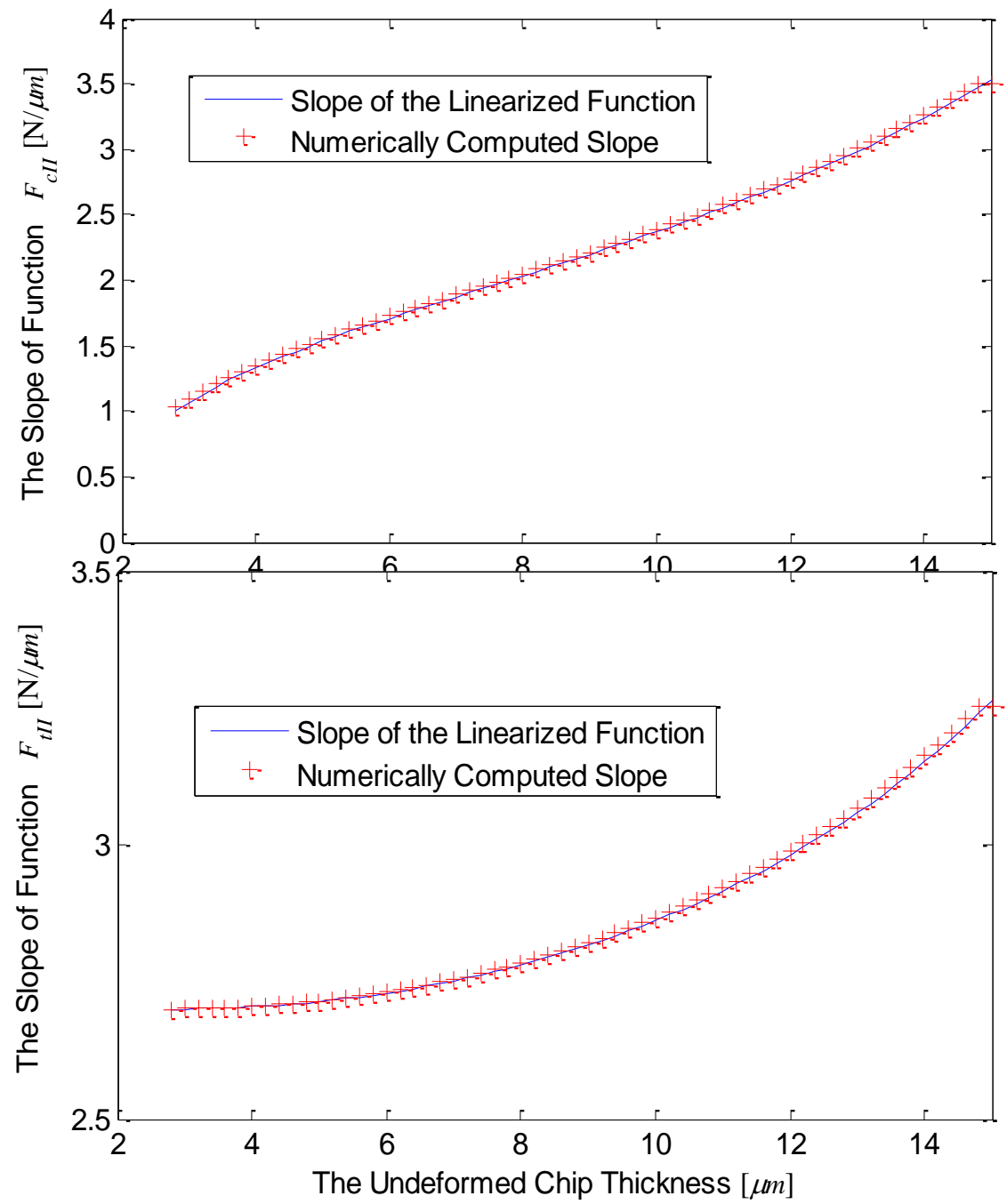

(b)

Fig. 3: Comparison of the Slopes of the Linearized Function with the Numerically Computed Slope of the (a) Cutting Force and (b) Tangential Force for $h_{m}<h<r_{t}$

Given that the stability analysis of a nonlinear system is very involved and that there is very little insight available into the stability of micro-cutting operations, even an initial qualitative understanding of the influence of the key process parameters on its stability would offer a first glance at the dominant issues. In this respect, in this paper the dynamic stability of the process will be examined based on a cutting dynamics process model that utilizes the linearized form of the proposed micro-cutting force model, discussed above, in combination with a simple linear structural dynamics model. The major advantage of this approach lies in the fact that linear 
stability theory can be used that, in turn, will facilitate a rigorous parametric study of the major factors that influence stability in light of the nonlinear phenomena that dominate the microcutting process.

\subsection{Micro-cutting Stability Criteria based on the Linearized Force Model}

In accordance with the classical linear cutting dynamics modeling approach, the structural dynamics model and the linearized force model will be combined to build the dynamic model for micro-cutting operations. The resulting characteristic equation will subsequently be analyzed to identify the critical axial depths of cut for different cutting conditions. As a first step in developing the stability criteria, the linearized function of the force model was developed. It was shown in the previous section that the cutting force for the different micro-cutting regimes can be, in general, expressed as the sum of a $0^{\text {th }}$ - and a $1^{\text {st }}$-order term of the undeformed chip thickness, i.e., as:

$$
F(t)=F_{\text {const }}+K_{f} a h(t)
$$

where $F_{\text {const }}$ is the static component of the cutting force and $K_{f}$ is the cutting coefficient, while $a$ and $h$ represent the width of cut and the undeformed chip thickness, respectively. In this case, the block diagram of the process is shown in Fig 4.

From the diagram, the output for the closed-loop system, in terms of the Laplace operator, assumes the well-known form:

$$
y(s)=\frac{K_{f} a \Phi(s)}{1+\left(1-e^{-s T}\right) K_{f} a \Phi(s)} h_{0}+\frac{F_{\text {Const. }} \Phi(s)}{1+\left(1-e^{-s T}\right) K_{f} a \Phi(s)}
$$

where $\Phi(s)$ is the linear lumped parameter structural dynamics model and T the process delay. 


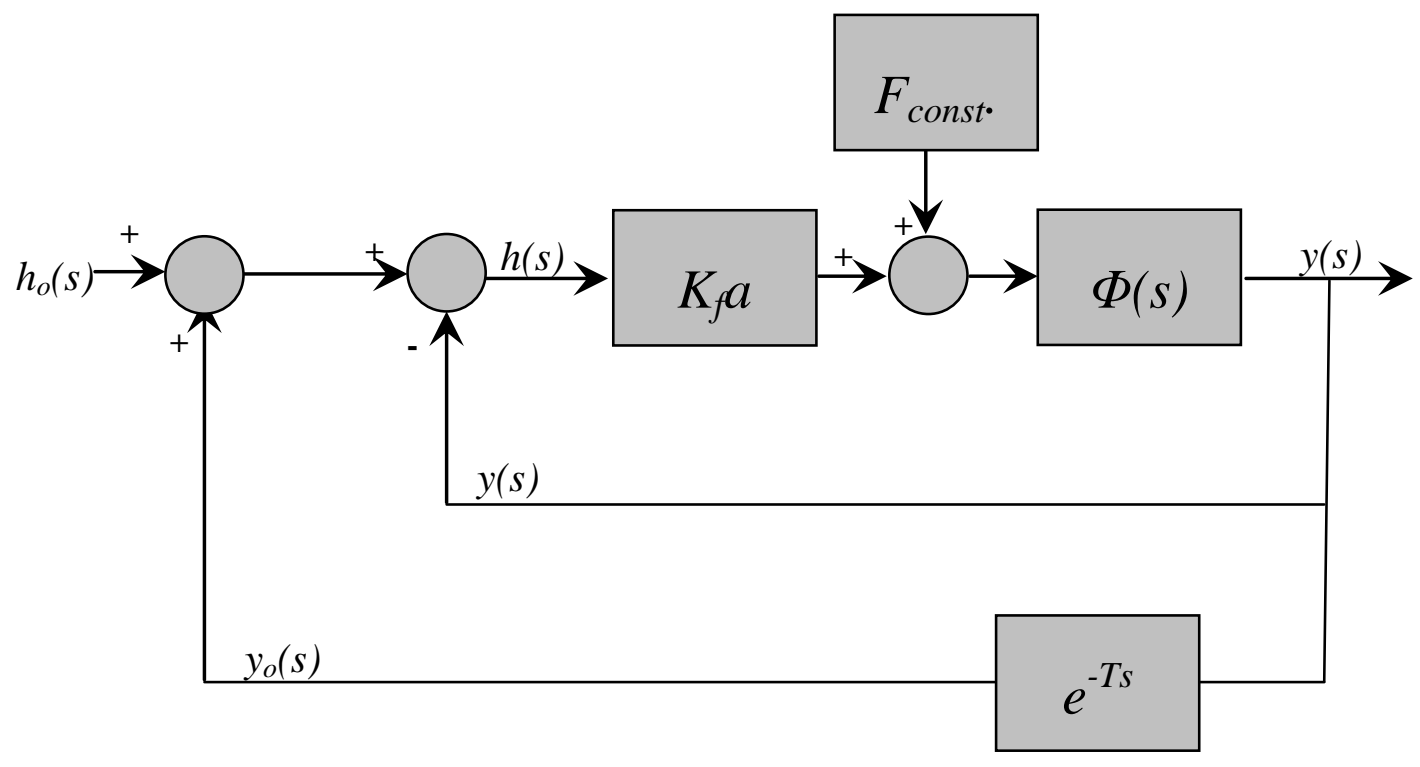

Fig. 4: Modified Block Diagram of Chatter Dynamics

Assuming that the second term on the right-hand side of Eq. (44) is a noise term, the characteristic equation of the transfer function becomes:

$$
1+\left(1-e^{-s T}\right) K_{f} a \Phi(s)=0
$$

This equation is identical to the characteristic equation of the classical regenerative linear chatter model, e.g., [6, 7]. The static component of the cutting force does not affect the characteristic equation based on the fact that the static component of the cutting force is time invariant and does not affect the dynamics of the process. Among the various criteria for chatter in machining, including Pontriagrin's theorem, D-subdivision method, and the Nyquist criterion, in this paper the latter, first introduced by Merritt [8], will be used.

Under the assumption of a single degree-of-freedom machine tool structural model:

$$
\Phi(j \omega)=G+j H
$$

and by considering the linearized process model in Eq. (43), the stability criteria can be derived in the usual form, i.e., Altintas [20]: 


$$
\begin{aligned}
& a_{\lim }=\frac{-1}{2 K_{f} G\left(\omega_{c}\right)} \\
& n=\frac{\omega_{c}}{2 i \pi+3 \pi+2 \xi}
\end{aligned}
$$

where $i=1,2,3, \ldots$ denotes the integer number of the wave on the surface and $\xi$ denotes the phase angle of the transfer function of the structure, i.e.:

$$
\xi=\tan ^{-1} \frac{H\left(\omega_{c}\right)}{G\left(\omega_{c}\right)} .
$$

By plotting the critical axial depth of cut vs. the spindle speed, the usual stability lobe diagrams can be constructed. When the force follows a nonlinear function, as in the current case, it should be expressed in a linearized form in order to apply the same approach. The proportionality constant in the linearized function is the specific cutting pressure and it indicates the amount of the dynamic force exerted on the structure. Therefore, the derivation of suitable expressions for the specific cutting pressure from the nonlinear function is the main issue in dealing with a nonlinear process model in dynamic stability analysis based on linear stability theory. In the current case, stability diagrams will not be developed for the three cases, since the procedures are well-known. Instead, the focus will be shifted to the discussion of the implications of the peculiar nature of the micro-cutting process on its stability. In this regard, in the current context, the meaning of the "cutting coefficient" in Eq. (43) should be carefully examined, for the cutting coefficient in the developed micro-cutting force model is profoundly different from that implied by the classical macro-scale linear force models.

\subsection{Specific Cutting Pressure in the Case of Nonlinear Process Functions}

The specific cutting pressure in the conventional linear force model is constant. However, in the proposed force model it is not constant but is a function of the undeformed chip thickness. Comparing Eq. (43) to Eq. (4a), the cutting coefficient can be expressed as: 


$$
K_{f}=\frac{d F_{c}}{d h}\left(h_{0}\right), \text { for the cutting direction }
$$

Figure 5, based on the linearized relationships for the three cases, derived in Section 3, shows the simulated values of the cutting force (dashed line) and of the specific cutting pressure (dotted line) as a function of the undeformed chip thickness for an assumed shear strength of the material of $170 \mathrm{MPa}$, tool edge radius $r_{t}$ of $15.0 \mu \mathrm{m}$ and a friction coefficient of 0.35 . The result clearly shows the total nonlinearity of the cutting force and of the corresponding specific cutting pressure. Not only the nonlinearity, but also discontinuities in the cutting force and in the specific cutting pressure can be observed in the figure. The discontinuity of the cutting force and of the specific cutting pressure raises a complex issue in the nonlinear dynamics analysis of the process, for the first derivative of the force function is indeterminate or not defined at the points of discontinuity.

Previous researchers who studied the nonlinearity of the cutting force model in chatter analysis dealt only with nonlinear force functions without discontinuities in their first derivatives. However, the force function and its derivatives, proposed in this paper, are discontinuous and the approaches used by previous researchers are no more valid. Therefore, a new approach to dealing with the nonlinear force function with discontinuities will be proposed and it will be discussed in more detail below.

Table 2 classifies the force function in terms of its linearity and continuity. The individual cases will be examined and a general methodology will be presented that is focused on the issue of how to deal with the nonlinear force function when it is applied in the framework of linear stability theory. When the first derivative is not continuous or not determined, ideas other than those that use the first derivative to express the specific cutting pressure must be considered. The acceptance of the first derivative for the specific cutting pressure will be again examined to develop a dynamic stability model with better prediction accuracy. 
Table 2: Classification of the Force Function in Linear Stability Theory

\begin{tabular}{|c|c|c|c|c|}
\hline & \multirow{2}{*}{ Linearity } & \multicolumn{2}{|c|}{ Continuity } & \multirow{2}{*}{ Remarks } \\
\hline & & $F_{c}$ & $\frac{d F_{c}}{d h}$ & \\
\hline Case 1 & Linear & $\mathrm{O}$ & $\mathrm{O}$ & $\begin{array}{l}\text { Conventional machining } \\
\text { range }\end{array}$ \\
\hline Case 2 & Nonlinear & O & 0 & $h<h_{m}, h_{m}<h<r_{t}, h>r_{t}$ \\
\hline Case 3 & Nonlinear & 0 & $x$ & $h=r_{t}$ \\
\hline Case 4 & Nonlinear & X & $x$ & $h=h_{m}$ \\
\hline
\end{tabular}

' $\mathrm{O}$ ' indicates continuity and ' $\mathrm{X}$ ' indicates discontinuity of a function.

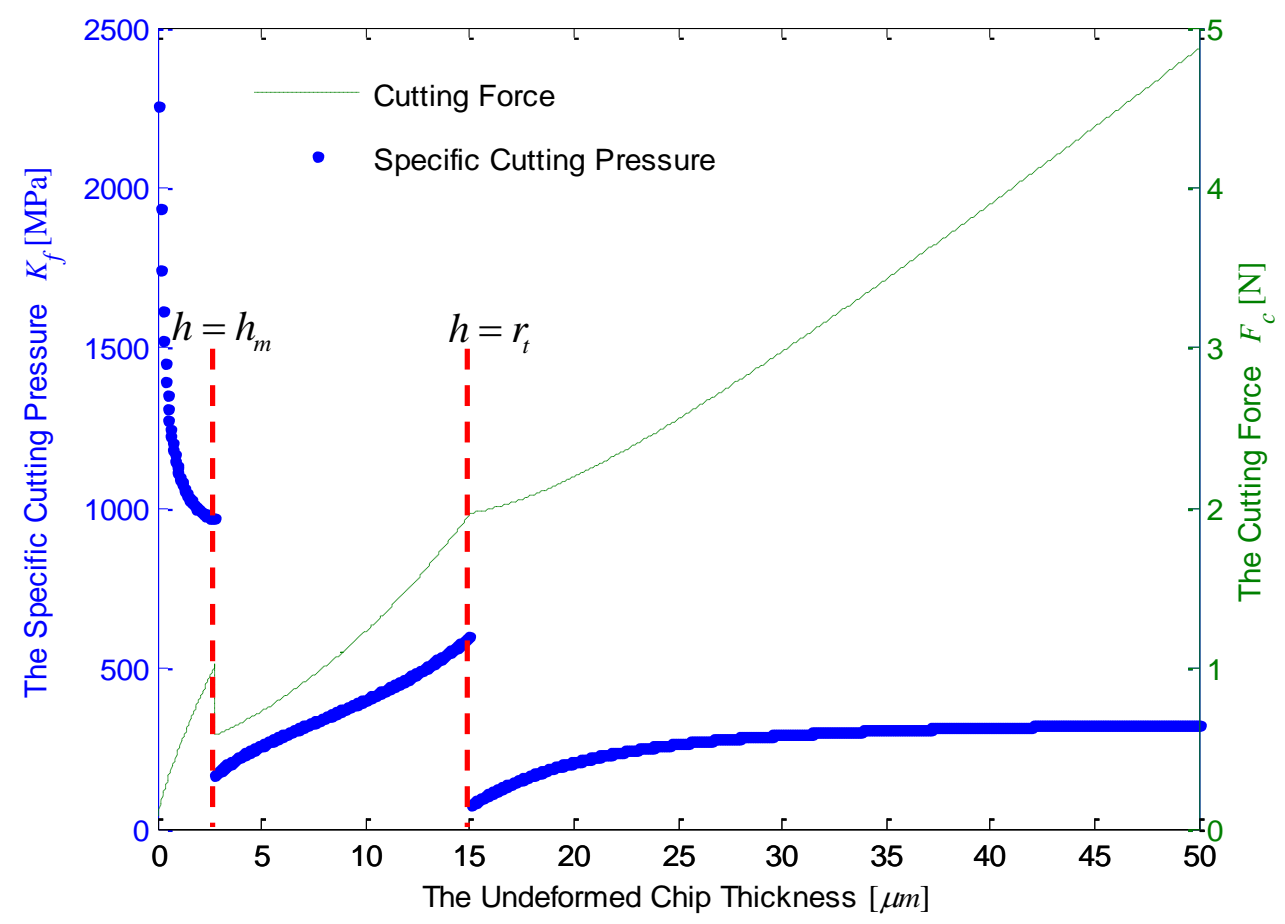

Fig. 5: Nonlinearity of the Cutting Force and of the Specific Cutting Pressure 


\subsubsection{Case 1: Linear Force Function}

When the force is linearly proportional to the undeformed chip thickness, the proportionality constant represents the specific cutting pressure. In this case the specific cutting pressure is invariant with respect to the undeformed chip thickness and the linear force equation can be directly applied. In Fig. 5, the cutting force is approximately linear and hence the specific cutting pressure converges to a constant value when the undeformed chip thickness is larger than about $40 \mu \mathrm{m}$. This situation corresponds to larger values of the undeformed chip thickness, i.e., conditions that characterize conventional machining processes.

\subsubsection{Case 2: Nonlinear and Differentiable Force Function}

When the force function is differentiable in the range of interest, the first derivative of the force function can be regarded as the specific cutting pressure, and expressed by:

$$
K_{f}=\frac{d F_{c}}{d h}\left(h_{0}\right)
$$

This is valid only when the change in the specific cutting pressure is negligible and can be ignored. However, the change in the specific cutting pressure in some ranges is not small enough to be neglected. As an example, the specific cutting pressure near $h=10.0 \mu m$ is shown in Fig. 6. Even with a small change in the undeformed chip thickness, a drastic change in the specific cutting pressure takes place. Therefore, the assumption of a constant specific cutting pressure should be again examined to assure the accuracy of the linearized force model.

From Fig. 6 it can be seen that the change in the specific cutting pressure is linear with the undeformed chip thickness. Based on this, a variable specific cutting pressure in the form of a linear function of the undeformed chip thickness can be introduced and its validity examined. To clarify the terminology to be used, the specific cutting pressure expressed by Eq. (50) will be referred to as constant specific cutting pressure of Type I.

Another constant specific cutting pressure concept to be introduced below will be referred

to as constant specific cutting pressure of Type II. This newly introduced specific cutting pressure, represented by a linear function of the undeformed chip thickness, will also be referred to as a variable specific cutting pressure. 


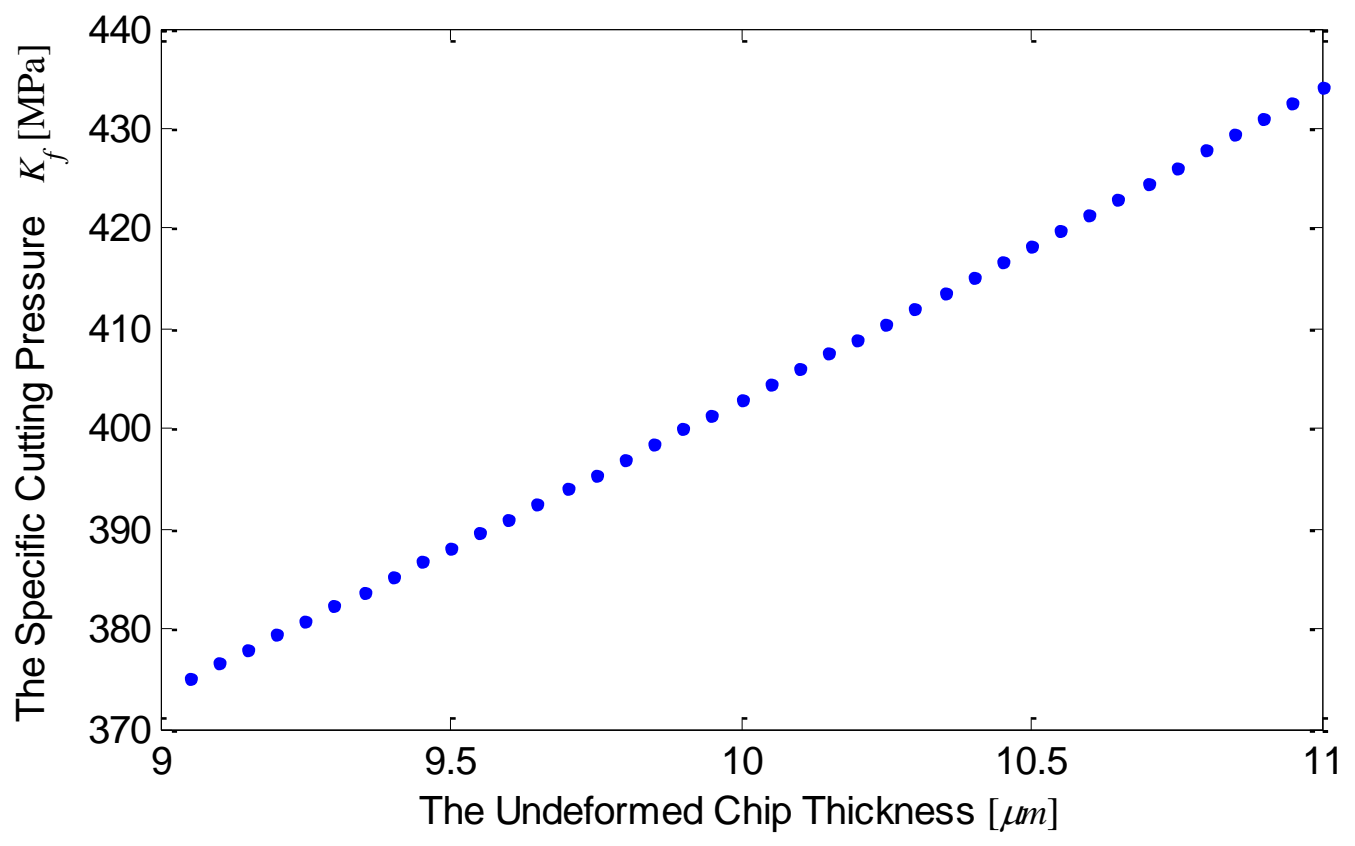

Fig. 6: Change in the Specific Cutting Pressure with Undeformed Chip Thickness

The variable specific cutting pressure is defined as a linear function of the undeformed chip thickness, i.e., as:

$$
\overline{K_{f}}(h)=\kappa_{0}+\kappa_{1} \cdot h, \quad h_{o}-\delta<h<h_{o}+\delta .
$$

where $\delta$ represents the admissible range within which the specific cutting pressure can be regarded as a linear function of the undeformed chip thickness. In this expression, $\kappa_{0}$ and $\kappa_{1}$ denote the $0^{\text {th }}$ and the $1^{\text {st }}$ order constants that represent the variable specific cutting pressure. Figure 7 shows the schematic of its identification from the first derivative of the force function. 


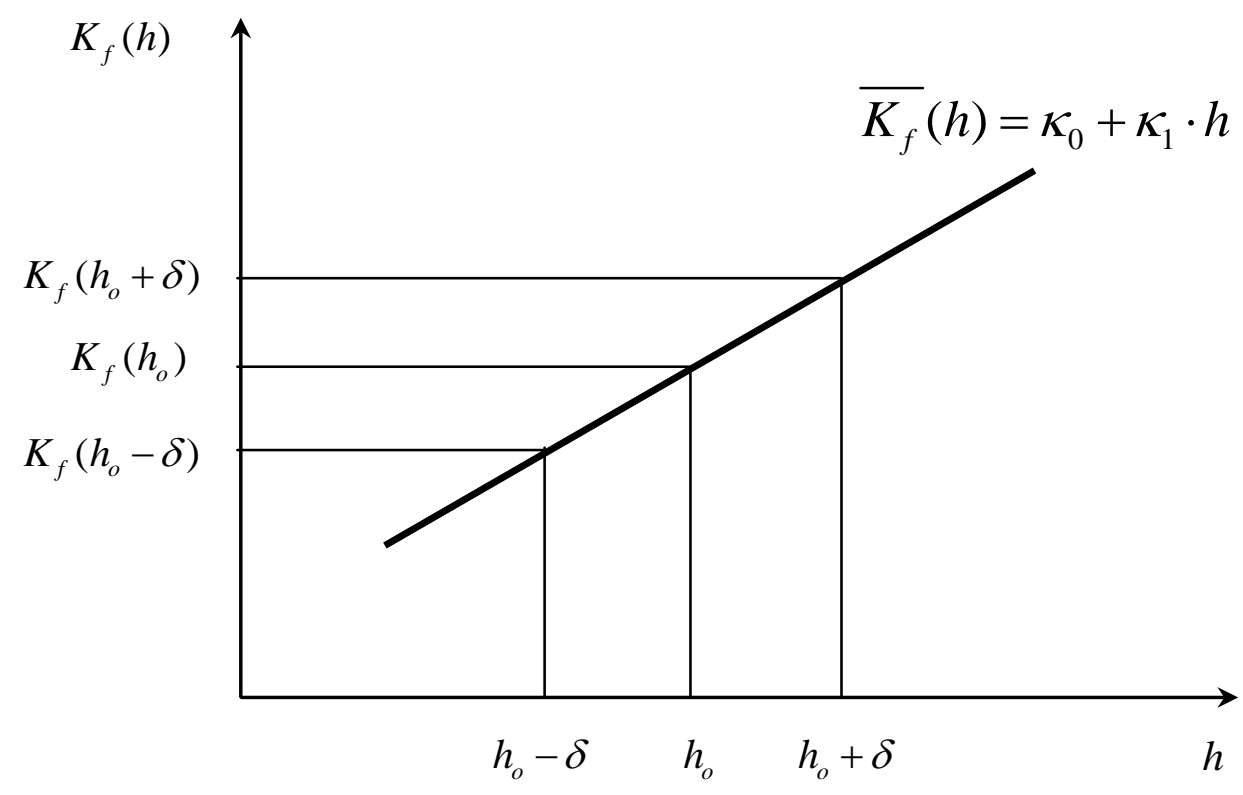

Fig 7: Variable Specific Cutting Pressure as a Linear Function of the Undeformed Chip Thickness when $h=h_{o}$

From Fig. 7, the linearly variable specific cutting pressure can be expressed by:

$$
\overline{K_{f}}(h)=\frac{K_{f}\left(h_{o}+\delta\right)-K_{f}\left(h_{o}-\delta\right)}{2 \delta}\left(h-h_{o}\right)+K_{f}\left(h_{o}\right)=\kappa_{0}+\kappa_{1} \cdot h .
$$

Therefore, the two constants, $\kappa_{0}$ and $\kappa_{1}$, can be written as:

$$
\begin{array}{ll}
\kappa_{0}=K_{f}\left(h_{o}\right)-\frac{K_{f}\left(h_{o}+\delta\right)-K_{f}\left(h_{o}-\delta\right)}{2 \delta} h_{o}, & h_{o}-\delta<h<h_{o}+\delta \\
\kappa_{1}=\frac{K_{f}\left(h_{o}+\delta\right)-K_{f}\left(h_{o}-\delta\right)}{2 \delta}, & h_{o}-\delta<h<h_{o}+\delta .
\end{array}
$$

To validate the variable specific cutting pressure concept, the predicted cutting force with the variable specific cutting pressure from Eq. (52) and the predicted cutting force with the constant cutting pressure from Eq. (50) are compared (Fig. 8). The reference force in Fig. 8 is calculated by the previously proposed nonlinear force model expressed by Eq. (2a). 


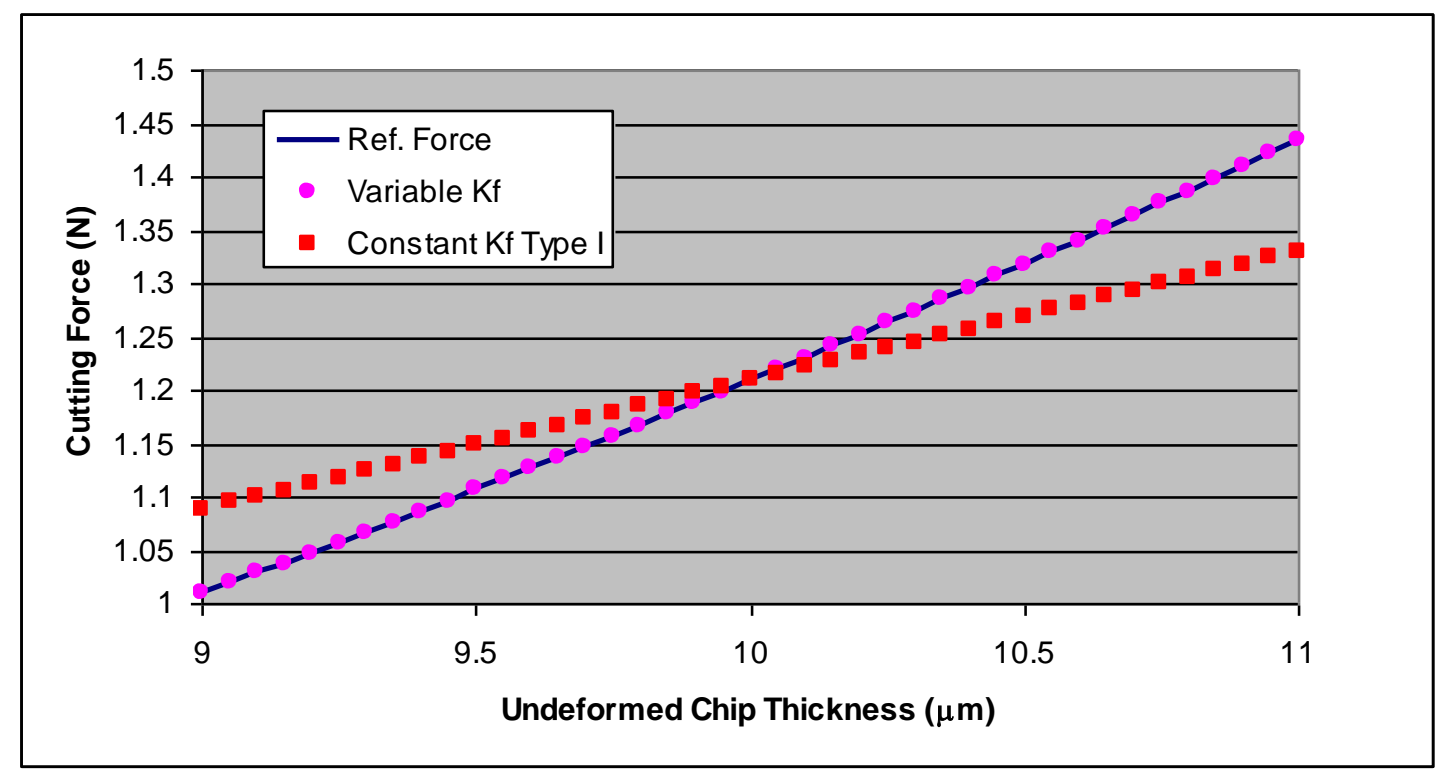

Fig 8: Comparison of the Cutting Force Prediction using Constant and Variable Specific Cutting Pressure Values

In Fig. 8, the force model with the variable specific cutting pressure represents the reference force model better than the force model with the constant specific cutting pressure of Type I. It is clear that the constant specific cutting pressure is valid if and only if the change in the specific cutting pressure is negligible and can be ignored. In the micro-cutting range, however, the change in the specific cutting pressure is not negligible even with a $1 \mu \mathrm{m}$ change in the undeformed chip thickness.

To predict the dynamic cutting force in a more precise way, a variable specific cutting pressure had to be introduced. In addition, the purpose of deriving a more suitable expression for the specific cutting pressure is also for its application in linear stability theory. However, a variable specific cutting constant cannot be directly applied in linear stability theory and a further linearization of the 'variable specific cutting constant' should be undertaken to complete the linear stability model. To this end, an expression for a constant specific cutting pressure of Type II will be derived from the variable specific cutting coefficient, for further use in linear stability analysis. In terms of the variable specific cutting pressure, the cutting force can be expressed by:

$$
F_{c}=\overline{K_{f}}(h) \cdot h=\kappa_{o} h+\kappa_{1} h^{2}, \quad h_{o}-\delta<h<h_{o}+\delta .
$$


The cutting force equation, Eq. (55), can be linearized by Taylor series expansion, leading to a linearized equation of the form:

$$
F_{c}(h)=\left(\kappa_{o} h_{o}+\kappa_{1} h_{o}^{2}\right)+\left(\kappa_{o}+2 \kappa_{1} h_{o}\right) \cdot\left(h-h_{o}\right), \quad h_{o}-\delta<h<h_{o}+\delta
$$

in which $\left(\kappa_{o}+2 \kappa_{1} h_{o}\right) \cdot h$ represents the dynamic component of the force that is linearly proportional to the undeformed chip thickness. To meet the above-stated requirement, the proportionality constant of the dynamic component of the force in Eq. (56) is being proposed to be taken as the constant specific cutting pressure of Type II, that is:

$$
\overline{\overline{K_{f}}}\left(h_{o}\right)=\kappa_{o}+2 \kappa_{1} h_{o}=K_{f}\left(h_{o}\right)+\frac{K_{f}\left(h_{o}+\delta\right)-K_{f}\left(h_{o}-\delta\right)}{2 \delta} h_{o} .
$$

The first term on the right side of Eq. (57), is the constant specific cutting pressure of Type I. The constant specific cutting pressure of Type II contains an additional term, $\frac{K_{f}\left(h_{o}+\delta\right)-K_{f}\left(h_{o}-\delta\right)}{2 \delta} h_{o}$ in comparison to the constant specific cutting pressure of Type I. The purpose of this modification of the constant specific cutting pressure is to achieve a more precise prediction of the dynamic cutting force than by the use of the specific cutting pressure constant of Type I. The linearized force based on the constant specific cutting pressure of Type II, expressed by Eq. (56), can be simplified to:

$$
F_{c}=\overline{\overline{K_{f}}}\left(h_{o}\right)\left(h-h_{o}\right)+K_{f}\left(h_{o}\right) \cdot h_{o}
$$

To validate the introduction of the variable specific cutting pressure and of the associated constant specific pressure of Type II, the cutting force was simulated and compared to a simulated force evaluated by using the constant specific cutting pressure of Type I. All the simulation results, including the proposed nonlinear force model denoted as the reference force, are shown in Fig. 9. The evaluations in Fig. 9 were again performed based on Eq. (2a) for the reference force, Eq. (50) for the linearized force model with the constant specific cutting pressure 
of Type I, Eq. (55) for the linearized force model with the variable specific cutting pressure, and Eq. (58) for the linearized force model with the constant specific cutting pressure of Type II, respectively. In the simulations $h_{o}$ and $\delta$ were set to $10.0 \mu \mathrm{m}$ and $1 \mu \mathrm{m}$, respectively. Note that Fig. 9 differs from Fig. 8 only by the addition of the simulation result for the linearized force with the constant specific pressure of Type II.

From the simulation results, it is evident that the variable specific cutting pressure and the constant specific cutting pressure of Type II represent the nonlinear force model more accurately than the constant specific cutting pressure of Type I. Therefore, the constant specific cutting pressure of Type II will represent the actual dynamic cutting force better than the constant specific cutting pressure of Type I when the linearized force model is applied in nonlinear stability theory.

Whether it is a Type I or II, the constant specific cutting pressure was derived from the first derivative of the force function when the function and its derivatives are well defined and continuous. In the case when the derivative of the function is not continuous, a different approach should be taken to acquire the specific cutting constant.

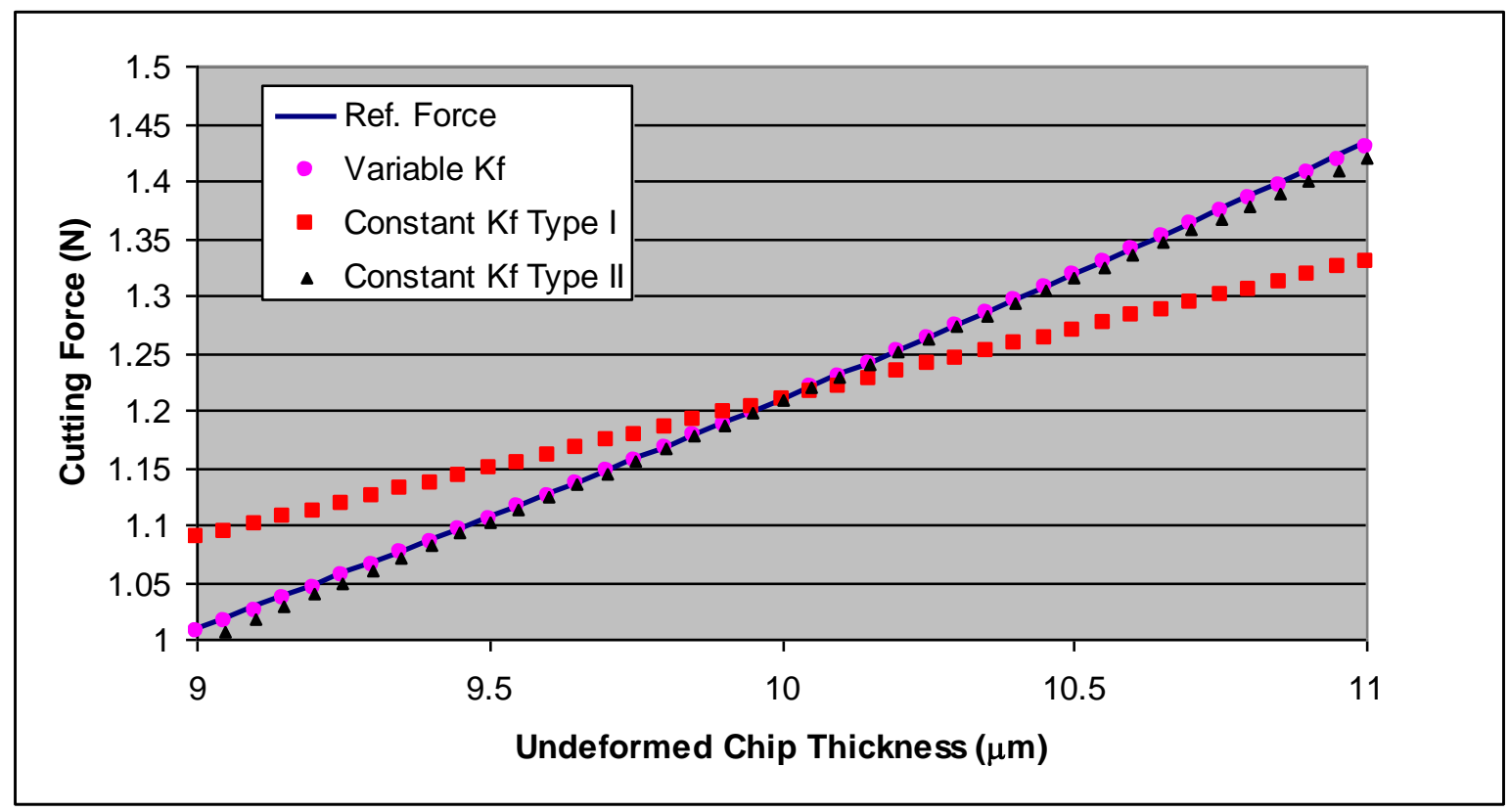

Fig. 9: Comparison of Cutting Force Predictions using Various Specific Cutting Pressures 


\subsubsection{Case 3: Nonlinear Force Functions with Discontinuous First Derivatives}

In Fig. 5, the specific cutting pressure is discontinuous but the force is continuous at $h=r_{t}$. In this case, there are two specific cutting pressure values of interest: $K_{f-}$ represents the limit from the left, while $K_{f+}$ represents the limit from the right. Figure 10 shows the discontinuity in the first derivative and the two limit values.

It is assumed here that the specific cutting pressure at any point can be written as:

$$
K_{f}=\frac{1}{2} \lim _{d x \rightarrow 0+} \frac{F_{c}(x+d x)-F_{c}(x)}{d x}+\frac{1}{2} \lim _{d x \rightarrow 0-} \frac{F_{c}(x+d x)-F_{c}(x)}{d x}
$$

Equation (59) is valid only when both the left-hand and the right-hand limits exist. Once the existence of both limits is confirmed, the cutting pressure can be written as:

$$
K_{f}=\frac{K_{f+}+K_{f-}}{2}
$$

The necessary condition to satisfy the existence of both limits is that the force is defined and continuous. Without continuity of the force function, Eq. (59) is not defined.

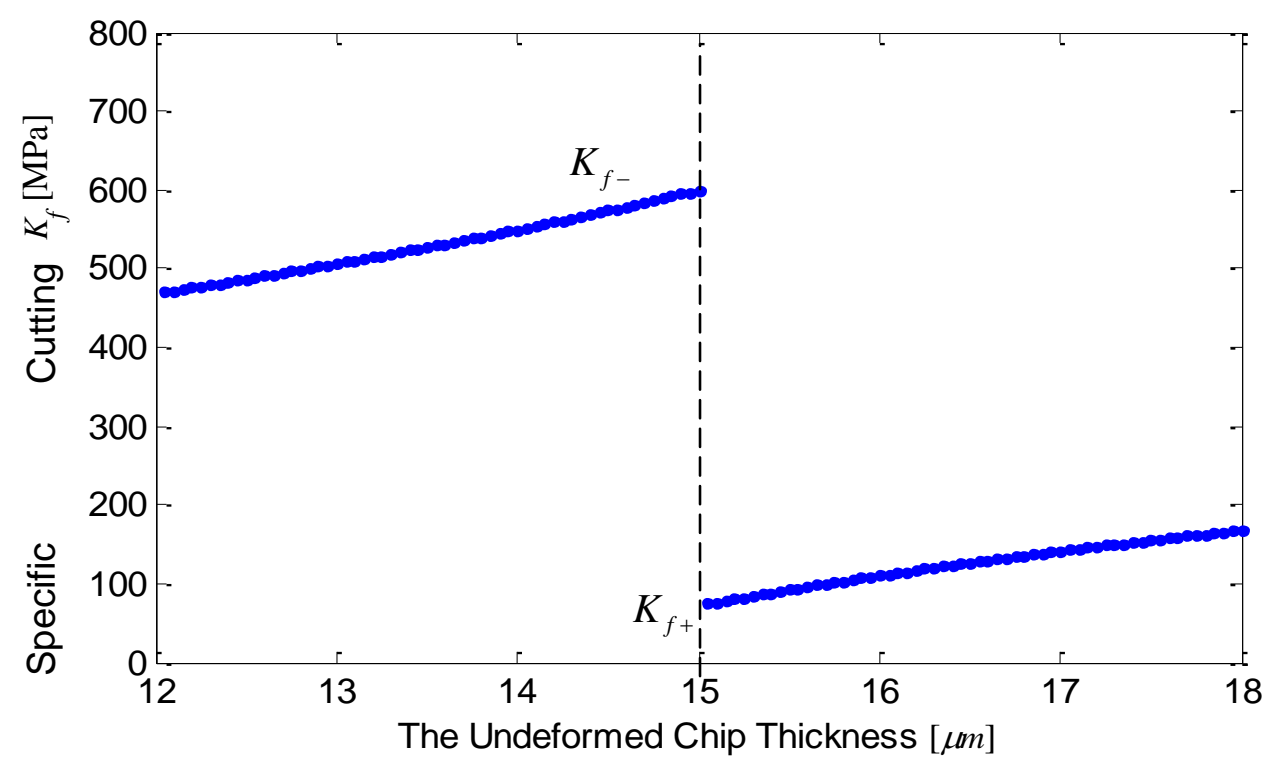

Fig. 10: Discontinuity in the First Derivative of the Force Function 


\subsubsection{Case 4: Nonlinear Discontinuous Force Function}

When the function is not defined at a certain point, no analytical expression of the specific cutting pressure can be formulated. In Fig. 5, there is a discontinuity in the cutting force when the undeformed chip thickness is equal to the minimum chip thickness. Therefore, the previously proposed methods are not valid and additional considerations should be made to account for the discontinuity in the force at this point. In the cutting force model proposed in this paper, the change of the force is stepwise and the first derivative is indeterminate when the undeformed chip thickness becomes equal to the minimum chip thickness. The step change in the cutting force at this point (dashed line in Fig. 11) as predicted by the respective expressions in Section 3 for Case I and Case II, under the same conditions as assumed in Fig. 5, was also experimentally validated (dotted line in Fig. 11) $[18,19]$. However, a closer examination of the experimental results reveals that the change in the force is not quite as abrupt, as predicted by the model, but rather it is gradual, although with a very steep gradient, indicating a smooth transition in the cutting mechanism. Hence, considering the physical reality confirmed by the experimental data, it is proposed to use the slope of the force data in the vicinity of the minimum chip thickness value as the specific cutting pressure at this point. Figure 11 shows the schematic of how to determine the specific cutting pressure from the experimental data.

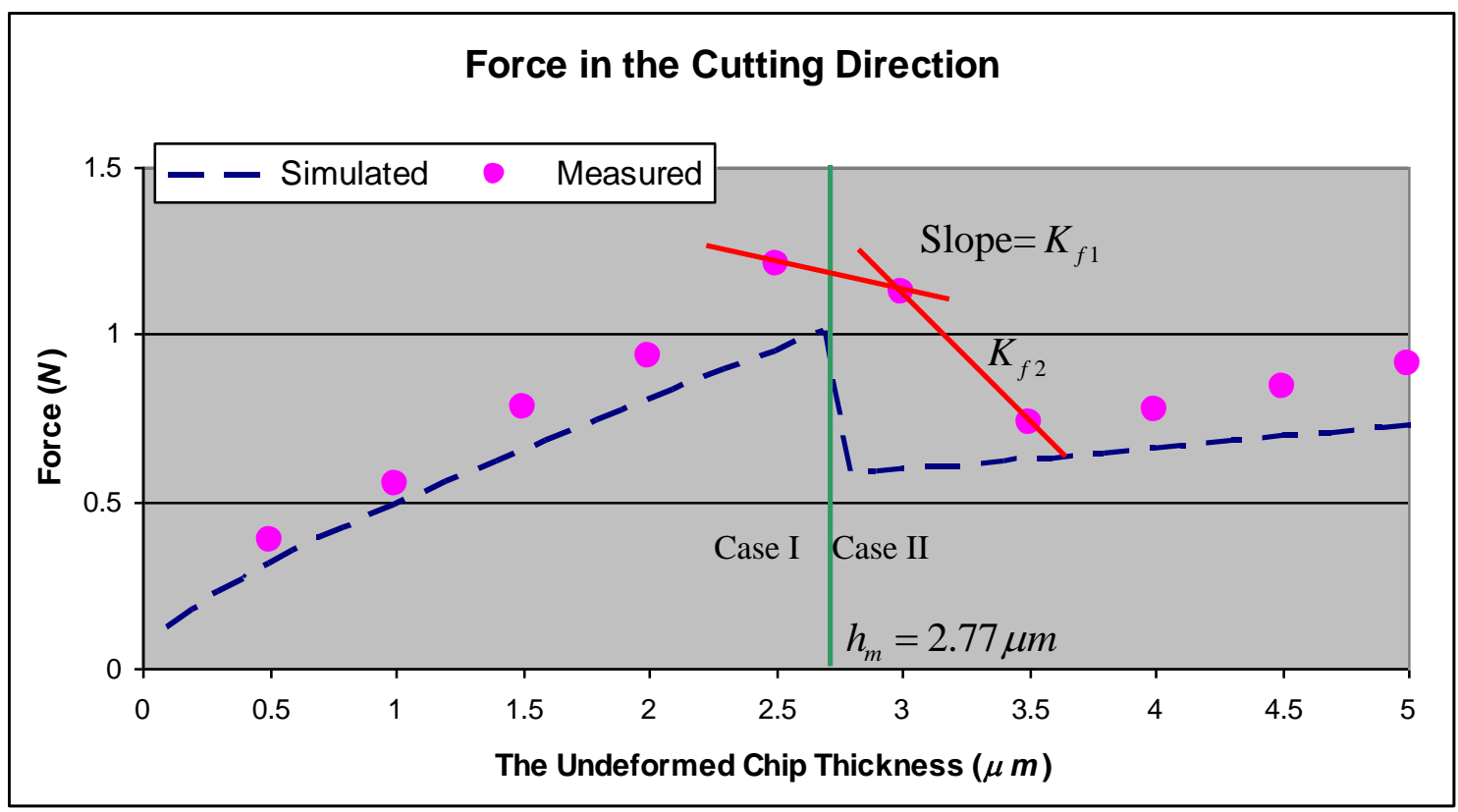

Fig. 11: Schematic of the Identification of the Specific Cutting Pressure at $h=h_{m}$ 
The slopes between the data points in the transition range can be calculated and the average of the slopes can be regarded as the specific cutting pressure at the minimum chip thickness value. In Fig. 11, there are three experimentally measured points in the transition range from which two slopes can be calculated and averaged. Table 3 summarizes the results of these calculations for the specific example.

Table 3: Calculated Specific Cutting Pressure at the Transition Point (MPa)

\begin{tabular}{|c|c|c|}
\hline$K_{f 1}$ & $K_{f 2}$ & $K_{f_{-} \text {Ave. }}$ \\
\hline \hline-520 & -2626 & -1573 \\
\hline
\end{tabular}

When the analytical function is not defined but experimental data is available, the numerical evaluation of the specific cutting pressure is a viable approach. When it comes to the numerical evaluation, there is one more issue to be considered: the resolution of the data. In the case presented above, only three data points can be found in the transition range and only two slopes can be extracted. It is evident that a higher resolution of the experimental data will lead to a higher accuracy of this approach and more experimental data should be acquired to increase the level of confidence. However, the resolution of the feed system limits the resolution of the experimental data. The resolution of the experimental data in this example, $0.5 \mu \mathrm{m}$, is considered very good for general micro-cutting research but a higher resolution would assure a higher level of statistical confidence of the measured data in the transition region.

\section{Summary and Discussion}

So far, a generalized approach, summarized in Table 4, for the evaluation of the specific cutting constant under various conditions of the force functions was discussed. It should be noted that the purpose of evaluating the specific cutting pressure is to utilize the linearized force equations for dynamic stability analysis in which the specific cutting pressure should be invariant with respect to the undeformed chip thickness if linear stability theory is to be applied. 
Table 4: The Specific Cutting Pressure for Different Nonlinear Force Functions

\begin{tabular}{|c|c|c|c|c|}
\hline & \multirow{2}{*}{ Linearity } & \multicolumn{2}{|c|}{ Continuity } & \multirow{2}{*}{ Specific Cutting Pressure } \\
\hline & & $F_{c}$ & $\frac{d F_{c}}{d h}$ & \\
\hline Case 1 & Linear & $\mathrm{O}$ & $\mathrm{O}$ & $K_{f}$ is explicitly expressed. \\
\hline Case 2 & Nonlinear & $\mathrm{O}$ & $\mathrm{O}$ & $\begin{array}{l}K_{f}\left(h_{o}\right): \text { Type I - Eq. (50) } \\
\overline{\overline{K_{f}}}\left(h_{o}\right): \text { Type II - Eq. }(57)\end{array}$ \\
\hline Case 3 & Nonlinear & $\mathrm{O}$ & $X$ & $K_{f}=\frac{K_{f+}+K_{f-}}{2}-$ Eq. (60) \\
\hline Case 4 & Nonlinear & $\mathrm{X}$ & $\mathrm{X}$ & Numerical Evaluation from Data \\
\hline
\end{tabular}

A generalized approach to the evaluation of the specific cutting pressure from the nonlinear cutting force model was discussed, as well as a new constant specific cutting pressure, named constant specific cutting pressure of Type II and expressed by Eq. (57), was proposed to more accurately predict the dynamic cutting force. Under these considerations, stability lobe diagrams can be constructed to predict the stable regions of micro-cutting processes. The stability lobe diagrams should be constructed separately for different undeformed chip thicknesses due to the specific cutting pressures and different continuity conditions of the force function and its derivative. To confirm the stability lobes, time-domain simulations of chatter were performed using Simulink developed by MathWorks. The Simulink model used for the simulation of regenerative chatter is shown in Fig. 12. An example of a stability lobe diagram is shown in Fig. 13. 


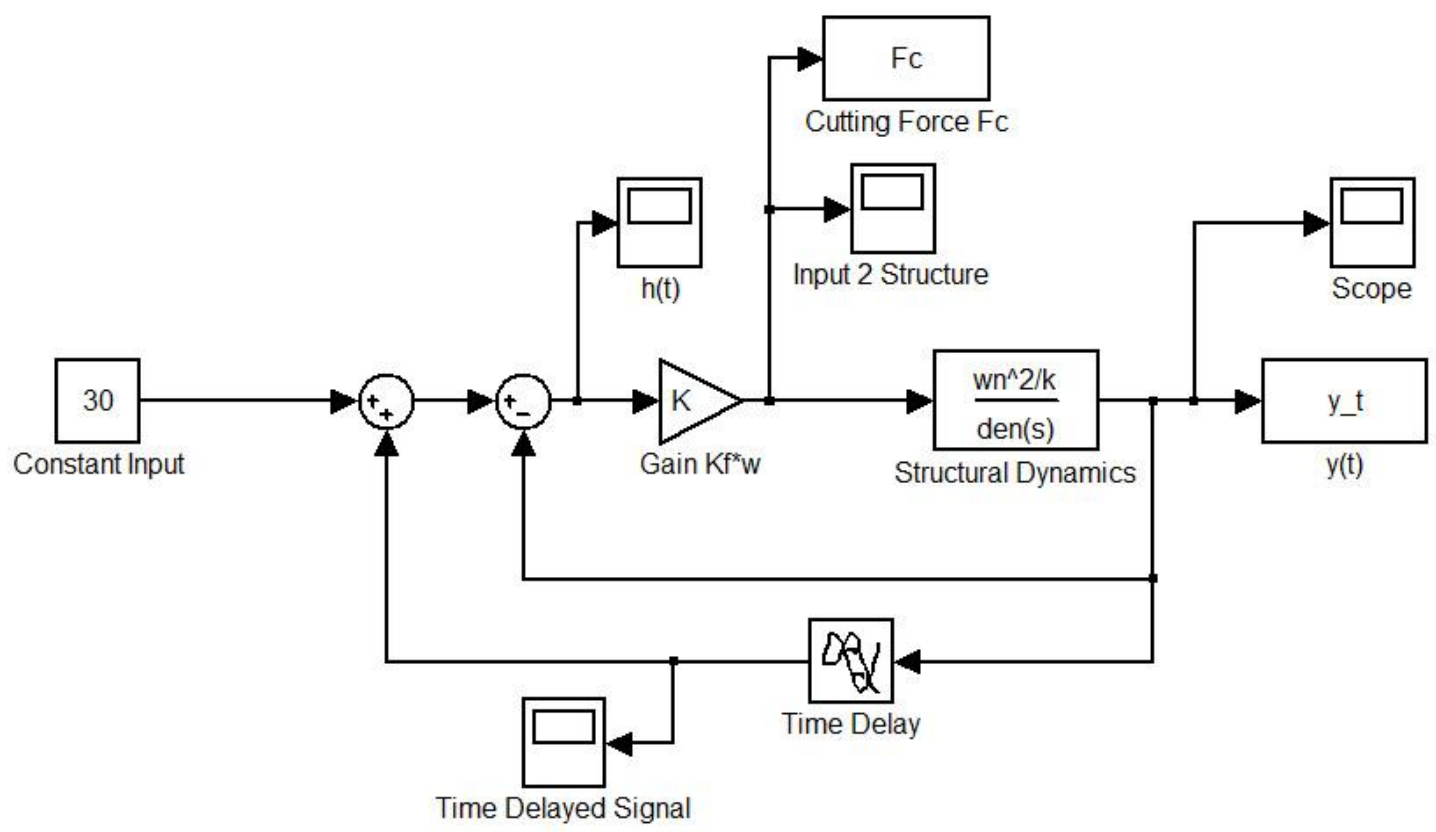

Fig. 12: Simulink Design for the Regenerative Chatter Simulator in the Continuous Time Domain

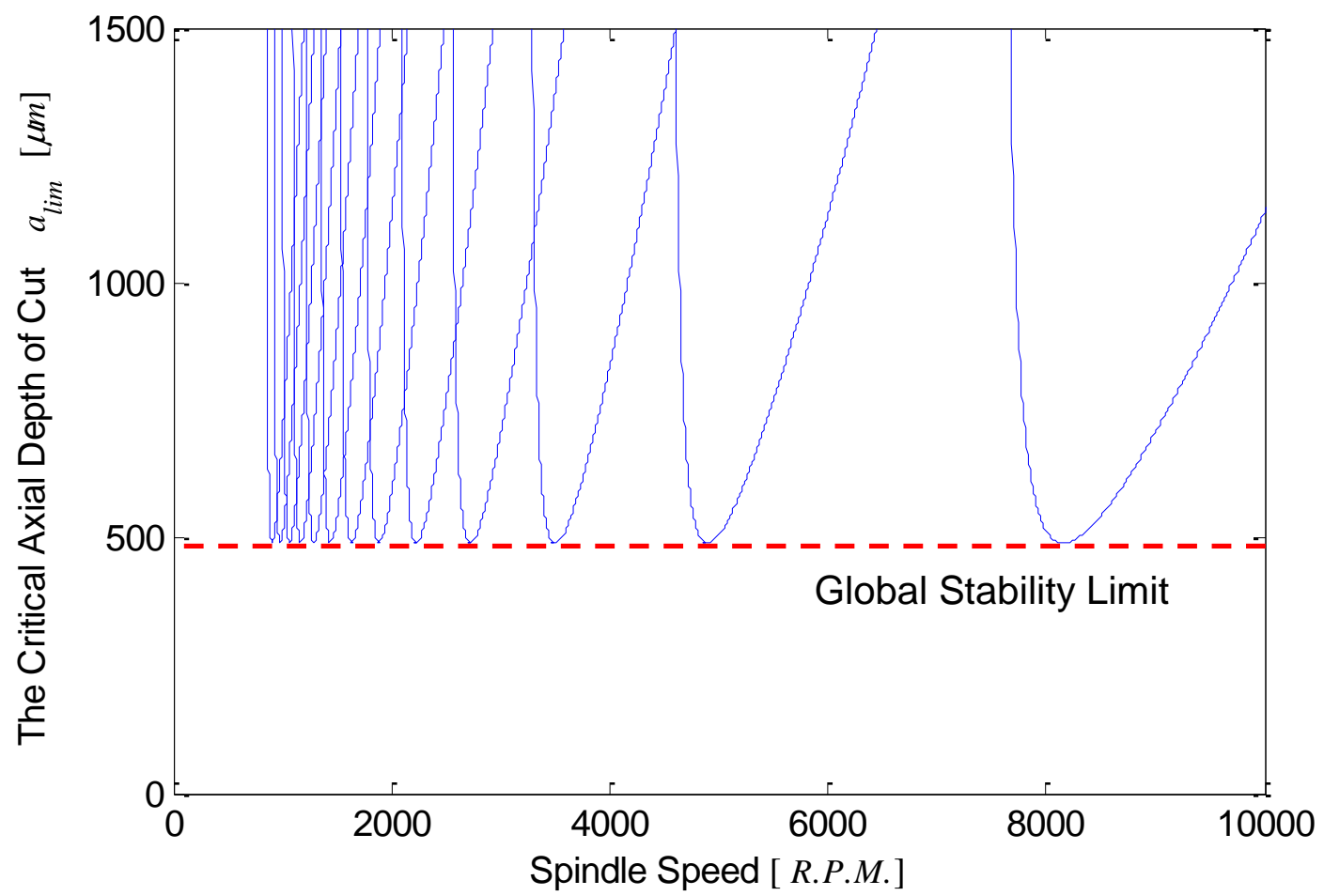

Fig. 13: Stability Lobes with Constant Specific Cutting Pressure Type I for $h=30.0 \mu \mathrm{m}$ 


\section{Conclusions}

The objective of this paper was to improve the fundamental understanding of the dynamics of the micro-cutting process as well as of its dynamic stability under the influence of nonlinear phenomena, especially the nonlinear behavior of the cutting force. The dynamic micro-cutting process was represented as consisting of two simultaneously acting mechanisms, namely chip formation and ploughing both assumed to be plastic flow processes. As a first step, a microcutting process model, developed based on the slip-line field, was analyzed followed by the formulation of a linearized form of the dynamic force expressions. Three distinct micro-cutting regimes that depend on the relation between the undeformed chip thickness and the tool cutting edge radius were defined.

For dynamic stability analysis, linear stability theory was adopted to model regenerative chatter. The linearized force model and a linear structural dynamics model were integrated to formulate stability criteria for the different characteristic operating regimes encountered in micro-cutting operations. Based on these developments a generalized approach to dealing with nonlinear force effects was proposed. The method is applicable for cutting process nonlinearities at all scales if they are present.

\section{Acknowledgments}

This work was supported by the National Science Foundation under grant number DMI0600175 . 


\section{References}

1. Vogler, M. P., Liu, X., Kapoor, S. G., Devor, R. E., and Ehmann, K. F., 2002, "Development of Meso-Scale Machine Tool (mMT) Systems," Transactions of NAMRI/SME, (30), pp. 653-661.

2. Liu, X., Devor, R. E. and Kapoor, S. G., 2006, " An Analytical Model for the Prediction of Minimum Chip Thickness in Micromachining," Journal of Manufacturing Science and Engineering, 128(2), pp. 474-481.

3. Weule, H., Hüntrup, V., and Tritschler, H., 2001, "Micro-Cutting of Steel to Meet New Requirements in Miniaturization," CIRP Annals - Manufacturing Technology, 50(1), pp. 61-64.

4. Liu, X., Devor, R. E., Kapoor, S. G., and Ehmann, K. F., 2004, "The Mechanics of Machining at the Microscale: Assessment of the Current State of the Science," Journal of Manufacturing Science and Engineering, 126(4), pp. 666-678.

5. Jun, M. B. G., Liu, X., Devor, R. E., and Kapoor, S. G., 2006, "Investigation of the Dynamics of Microend Milling---Part I: Model Development," Journal of Manufacturing Science and Engineering, 128(4), pp. 893-900.

6. Tobias, S. A., 1965, Machine Tool Vibration. Blackie and Son Limited, J. Wiley, London.

7. Tlusty, J. and Polacek, M., 1963 "The Stability of Machine Tools Against Self-Excited Vibrations in Machining," International Research in Production Engineering, pp. 465-474.

8. Merrit, H. E., 1965, "Theory of Self-Excited Machine Tool Chatter," ASME Journal of Engineering for Industry, pp. 447-454.

9. $\quad$ Smith, S. and Tlusty, J., 1993, "Efficient Simulation Programs for Chatter in Milling," CIRP Annals - Manufacturing Technology, 42(1), pp. 463-466.

10. Minis, I. and Yanushevsky, R., 1993, "A New Theoretical Approach for the Prediction of Machine Tool Chatter in Milling," Journal of Engineering for Industry, 115(1), pp. 1-8.

11. Minis, I., Yanushevsky, R., Tembo, A., and Hocken, R., 1990, "Analysis of Linear and Nonlinear Chatter in Milling," CIRP Annals - Manufacturing Technology, 39(1), pp. 459462.

12. Altintas, Y., and Budak, E., 1995, "Analytical Prediction of Stability Lobes in Milling," CIRP Annals - Manufacturing Technology, 44(1), pp. 357-362.

13. Hanna, N. H., and Tobias, S. A., 1974, "A Theory of Nonlinear Regenerative Chatter," Transactions of the American Society of Mechanical Engineers - Journal of Engineering for Industry, 96(1), pp. 247-255. 
14. Moon, F. C., Kalmár-Nagy, T., 2001, "Nonlinear Models for Complex Dynamics in Cutting Materials," Philosophical Transactions of the Royal Society of London. Series A: Mathematical, Physical and Engineering Sciences, 1781(359), pp. 695-711.

15. Stépán, G., 2001, "Modelling Nonlinear Regenerative Effects in Metal Cutting," Philosophical Transactions of the Royal Society of London. Series A:Mathematical, Physical and Engineering Sciences, 1781 (359), pp. 739-757.

16. Jun, M. B. G., Devor R. E., and Kapoor, S. G., 2006 "Investigation of the Dynamics of Microend Milling---Part II: Model Validation and Interpretation," Journal of Manufacturing Science and Engineering, 128(4), pp. 901-912.

17. Kim, C. J., Mayor, J. R., and Ni, J., 2004, "A Static Model of Chip Formation in Microscale Milling," Journal of Manufacturing Science and Engineering, 126(4), pp. 710-718.

18. Yoon, H. S., 2009, "Dynamics of the Micro-Machining Process," Ph.D. thesis, Northwestern University, Evanston.

19. Yoon, H. S., and Ehmann, K. F., Submitted, 2014, "Forces in Micro-Cutting Operations: Part I- Model Formulation; Part II- Model Verification," Journal of Manufacturing Science and Engineering.

20. Altintas, Y., 2000, Manufacturing Automation, Cambridge University Press.

21. Wiercigroch M., Budak E., 2001, "Sources of Nonlinearities, Chatter Generation and Suppression in Metal Cutting," Phil. Trans. The Royal Society of London A Mathematical Physical And Engineering Science, 359(1781):663-693.

22. Wiercigroch M., Krivtsov A. M., 2001, "Frictional Chatter in Orthogonal Metal Cutting," Phil. Trans. The Royal Society of London A Mathematical Physical And Engineering Science, 359(1781): 713-738. 